\title{
A Local Convergence Proof for the minvar Algorithm for Computing Continuous Piecewise Linear Approximations*
}

\author{
Richard E. Groff Pramod P. Khargonekar Daniel E. Koditschek
}

\begin{abstract}
The class of continuous piecewise linear (PL) functions represents a useful family of approximants because invertibility can be readily imposed, and if a PL function is invertible, then it can be inverted in closed form. Many applications, arising for example in control systems and robotics, involve the simultaneous construction of a forward and inverse system model from data. Most approximation techniques require that separate forward and inverse models be trained, whereas an invertible continuous PL affords, simultaneously, the forward and inverse system model in a single representation. The minvar algorithm computes a continuous PL approximation to data. Local convergence of minvar is proven for the case when the data generating function is itself a PL function and available directly rather than through data.
\end{abstract}

${ }^{*}$ A version of this manuscript will appear in the SIAM Journal on Numerical Analysis. This technical report contains proofs and details which are omitted in the SIAM version. This work is supported in part by NSF Award ECS-96322801 


\section{Introduction}

In this paper, we present minvar, a novel algorithm for computing continuous multidimensional piecewise linear (PL) approximations to data. The algorithm takes advantage of the structure of PL functions to provide a computationally effective approximation technique. This paper provides a local convergence proof for the special case that the data generating function is itself PL and is available directly rather than through discrete data.

Our interest in the PL family is driven by applications that require approximation of both forward and inverse functions from data. For example, in xerography, the print engine's color space transformation is required to stabilize color reproduction while its inverse is required to generate printer specific color mixture commands in response to inputs expressed in device independent color coordinates [21, 23]. The field of robotics is rife with examples where changes of coordinates play a key role: in mobile robot navigation $[27,33,34]$; in the representation of gaits [32, 35]; in sensor based manipulation [8]; as well as in calibration [43]. Since a change of coordinates is a continuous and continuously invertible function, building a custom change of coordinates amounts to a search for the appropriate forward and inverse function. Representations of scalar invertible functions are required for certain machine tool calibration problems [24], for certain automobile fuel control settings [17], as well as for probability density estimation[15]. In all such settings, most approximation techniques require the construction of distinct forward and inverse representations, because the approximations are not invertible in closed form. In addition to doubling the effective training effort, accuracy suffers since the approximation of the inverse is not exactly the inverse of the forward approximation. In contrast, invertibility of PL functions can be verified and even imposed geometrically, that is, by well characterized and computationally effective techniques arising from geometric insights. Moreover, if a PL function is invertible, it can be inverted in closed form. Thus a single PL approximation is ideal for applications requiring the approximation of a function and its inverse.

A substantial mathematical literature on real function approximation (see, for example, $[6,9,29])$, largely concerned with linear-in-parameters techniques, deals extensively with algorithms, fundamental limits, convergence rates, and families of bases in approximating functions. Recent activity has been spurred by evidence that nonlinear-in-parameters function families offer improved approximation rates in higher dimensions as compared to linear-in-parameters representations [3]. Recently, approximation methods that employ collections of local approximations have received increasing attention $[1,16,39]$. However, very little of this linearor nonlinear-in-parameters literature addresses the problem of function approximation under the constraint of invertibility.

Piecewise linear functions have been addressed in a number of different settings. Algebraic topologists used PL homeomorphisms to classify topological spaces [37], but did not address computational considerations. The study of splines, piecewise polynomials with continuity and smoothness constraints, includes piecewise linear functions $[7,10,36]$. Splines are typically extended to multiple dimensions by means of tensor products. The domain partition is then a tensor product of partitions of the individual dimensions and the approximant is the sum of tensor products of scalar spline functions. A multidimensional linear spline is then multilinear, that is linear in each variable separately, rather than truly linear. General splines enjoy no invertibility properties. Moreover, most of the spline literature assumes the domain partition to be fixed, in which case approximation of the best $L_{2}$ spline is a linear-in-parameters problem. Allowing the partition to change introduces a nonlinear-in-parameters problem. The Multivariate Adaptive Regression Spline (MARS) literature admits a limited nonlinear parameterization by allowing the basis to adapt, but does not allow general motion of the domain partition[16,39]. The piecewise polynomial literature addresses the problem of finding (possibly discontinuous) piecewise polynomial approximations to an explicitly known scalar function. In this setting, the domain partition is considered as part of the approximation's parameterization. For scalar functions, there are results for the existence of a best approximation by possibly discontinuous piecewise polynomials under certain generalized convexity conditions [4, 18]. Algorithms similar in flavor to the scalar specialization of minvar were introduced in $[2,25,26]$. A treatment of discontinuous piecewise polynomial approximations on two dimensional triangulations is provided in [40]. Also, [41] provides an algorithm for a moving mesh finite element solution to variational problems. A specialization of this moving 
mesh algorithm is finding the best $L^{p}, p$ finite and even, continuous piecewise polynomial approximation to a function. Both of these algorithms [40,41], as well as the piecewise polynomial literature in general $[2,25,26]$, assume that the function to be approximated is available directly, and the algorithms entail steps, such as root finding, that incorporate the function intrinsically. In contrast, the minvar algorithm is defined for arbitrary (finite) dimension and can either use a finite set of data or directly use of the function to be approximated.

Motivated by applications that require the approximation of invertible functions, we have developed the minvar algorithm for computing piecewise linear approximations to a set of discrete data. In the context of these applications, PL approximations offer the substantial benefit of closed form invertibility. When the domain partition is fixed, computing the best piecewise linear approximation is a linear-in-parameters problem that can be solved using classical techniques. Treating the partition as a component of the approximation's parameterization gives a much more powerful approximant, at the cost of entering the nonlinearin-parameters problem domain. In nonlinear-in-parameters problems, one can generally expect only local, as opposed to global, convergence properties. Moving the domain partition of a PL function, or triangulation as formally defined in the next section, has an added difficulty. A triangulation has both continuous and combinatorial parameters that interact in complex ways. Not all combinations of continuous and combinatorial parameters yield a proper triangulation. Triangulations in two and three dimensions have been studied extensively in the computational geometry literature [13, 31], but results for general dimension are more scarce, notwithstanding significant recent progress [5, 14, 28]. The price of using a family of finitely parameterized homeomorphisms, the PL approximations, is the cost of managing the combinatorial complexities of PL functions.

This paper is divided into five main sections. Section 2 provides a careful definition of the concept of a triangulation, relating it to the parameterization of PL functions. Section 3 introduces and defines the minvar algorithm. Section 4 provides a local convergence proof for the minvar algorithm when the data generating function is piecewise linear. Section 5 presents a numerical example.

\section{Triangulations and Piecewise Linear Functions}

The ability to check invertibility of a PL function, and to invert it in closed form, derives from the interplay between the PL's combinatorial and continuous parameters. This interplay provides much power, but also creates potential pitfalls. For example, changing the continuous parameters inappropriately with respect to the combinatorial structure can cause "tangles" in the domain partition. Triangulations in general dimension, the key concept in understanding PL functions, are still an area of active research in computational geometry. While the minvar algorithm can be stated using only an intuitive notion of triangulation, further analytical insight, such as the local convergence proof provided in section 4, is limited without a much more careful definition. This section provides definitions of triangulations and PL functions to facilitate the exposition. For further background, see [42] for an introduction to concepts in convexity, and [13] for an introduction to the geometric concept of triangulations.

\subsection{Simplices}

An affine subspace $V \subseteq \mathbb{R}^{d}$ is a linear subspace $L \subseteq \mathbb{R}^{d}$ translated by some $x_{o} \in \mathbb{R}^{d}$, i.e. $V=L+x_{o}$. The dimension of $V$ is $\operatorname{dim}(V):=\operatorname{dim}(L)$. The affine hull of a set $\mathcal{U} \subseteq \mathbb{R}^{d}$, aff $(\mathcal{U})$, is the smallest affine subspace containing $\mathcal{U}$. A finite set of points $\mathcal{U} \subseteq \mathbb{R}^{d}$ is affinely independent if for $i=1, \ldots, d$, no affine subspace of dimension $i$ contains more that $i+1$ points from $\mathcal{U}$. The convex $h u l l$ of a set $\mathcal{U} \subseteq \mathbb{R}^{d}, \operatorname{conv}(\mathcal{U})$, is the smallest convex set containing $\mathcal{U}$. A simplex, $s$, is the convex hull of a (finite) set $\mathcal{V} \subseteq \mathbb{R}^{d}$ of affinely independent points, $s=\operatorname{conv}(\mathcal{V})$. The set of the extreme points, or vertices, of $s, \mathcal{V}=\operatorname{vert}(s)$, uniquely defines $s .{ }^{1}$ The dimension of $s$ is $l=\operatorname{dim}(s)=\operatorname{dim}(\operatorname{aff}(s))=\operatorname{card}(\mathcal{V})-1$, where card $(\mathcal{V})$ is the cardinality of $\mathcal{V}$, and $s$

\footnotetext{
${ }^{1}$ vert is a pseudoinverse of conv, not a true inverse, since if $\mathcal{U} \subseteq s$, then $s=\operatorname{conv}(\mathcal{U} \cup \operatorname{vert}(s))$. The concept of vertices of a simplex is a special case of the more general notion of extreme points of a convex body. The Krein-Milman Theorem [42] states that any convex compact set in $\mathbb{R}^{d}$ is the convex hull of its extreme points.
} 


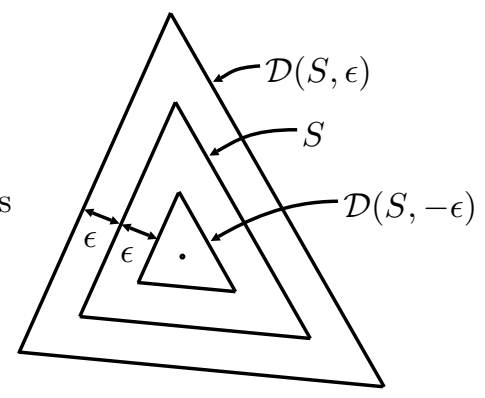

Figure 1: The 2-simplex $S$ with its $\epsilon$ and $-\epsilon$ dilations. The point at the center is where dilation degenerates to a single point.

is called an $l$-simplex. There can be at most $d+1$ affinely independent points in $\mathbb{R}^{d}$, and thus there are simplices of dimension $-1,0,1, \ldots, d$, where by convention $\emptyset$ is considered a simplex with $\operatorname{dim}(\emptyset):=-1$. We may apply a partial order to simplices. Given two simplices $s_{1}, s_{2}$, we say that $s_{1} \leq s_{2}$ if and only if $\operatorname{vert}\left(s_{1}\right) \subseteq \operatorname{vert}\left(s_{2}\right)$, in which case we call $s_{1}$ a face of $s_{2}$. If $\operatorname{dim}\left(s_{2}\right)=d$ and $\operatorname{dim}\left(s_{1}\right)=d-1$, then we also call $s_{1}$ a facet of $s_{2}$. In this paper we will predominantly be interested in $d$-simplices, so we adopt the convention that a capital $S$ indicates a $d$-simplex, while a lowercase $s$ denotes a simplex of any dimension.

Let $S$ be a $d$-simplex with $\operatorname{vert}(S)=\left\{p_{1}, \ldots, p_{d+1}\right\}$. The $\epsilon$ dilation of $S$, written $\mathcal{D}(S, \epsilon)$, is defined as

$$
\mathcal{D}(S, \epsilon):=\left\{x=\sum_{j=1}^{d+1} \alpha_{j} p_{j} \mid \sum_{j=1}^{d+1} \alpha_{j}=1, \forall j, \alpha_{j} \geq \frac{-\epsilon}{\delta\left(p_{j}, \operatorname{aff}\left(\operatorname{vert}(S)-\left\{p_{j}\right\}\right)\right)}\right\},
$$

where $\delta(p, \mathcal{U})$ is the distance from point $p$ to the nonempty set $\mathcal{U} \cdot \mathcal{D}(S, \epsilon)$ is well defined for

$$
\epsilon \geq-\left(\sum_{j=1}^{d+1} 1 / \delta\left(p_{j}, \operatorname{aff}\left(\operatorname{vert}(S)-\left\{p_{j}\right\}\right)\right)\right)^{-1}
$$

When equality holds, $\mathcal{D}(S, \epsilon)$ is a single point, otherwise it is a $d$-simplex with facets parallel to $S_{i}$, but distance $|\epsilon|$ away, with $S \subseteq \mathcal{D}(S, \epsilon)$ for $\epsilon>0$, and $\mathcal{D}(S, \epsilon) \subseteq S$ for $\epsilon<0$. (See Appendix A Claim 3 for the dilation's properties.)

\subsection{Triangulation}

An abstract simplicial complex is a collection of finite sets $\mathcal{S}$ satisfying if $\alpha \in \mathcal{S}$ and $\beta \subseteq \alpha$ then $\beta \in \mathcal{S}$. The vertex set of an abstract simplicial complex is the set $\{x \mid x \in \alpha, \alpha \in \mathcal{S}\}$.

A geometric simplicial complex is a collection $\mathcal{K}$ of simplices in $\mathbb{R}^{d}$ satisfying

1. $s_{1} \in \mathcal{K}$ and $s_{2} \leq s_{1} \Longrightarrow s_{2} \in \mathcal{K}$

2. $s_{1}, s_{2} \in \mathcal{K} \Longrightarrow s_{1} \cap s_{2} \leq s_{1}, s_{2}$.

The vertex set of a geometric simplicial complex is $\operatorname{vert}(\mathcal{K}):=\bigcup_{s \in \mathcal{K}} \operatorname{vert}(s)$. The underlying space of a geometric simplicial complex is $|\mathcal{K}|:=\bigcup_{s \in \mathcal{K}} s$.

A subcomplex is a subset of a simplicial complex that is itself a simplicial complex. The closure of a subset $\mathcal{L} \subseteq \mathcal{K}$ is the smallest subcomplex that contains $\mathcal{L}$,

$$
\mathrm{Cl} \mathcal{L}:=\{\alpha \in \mathcal{K} \mid \alpha \leq \beta, \beta \in \mathcal{L}\}
$$


The star of a simplex $s$ is the set of all simplices that contain $s$,

$$
\text { St } s:=\left\{s^{\prime} \in \mathcal{K} \mid s \leq s^{\prime}\right\}
$$

The star is not in general a subcomplex.

We can parameterize ${ }^{2}$ a geometric simplicial complex $\mathcal{K}$ in $\mathbb{R}^{d}$ by the pair $(P, \mathcal{S})$, where $P$ is an indexed set of $n$ unique points in $\mathbb{R}^{d}$,

$$
P=\left\{p_{1}, \quad p_{2}, \ldots, \quad p_{n}\right\},
$$

and $\mathcal{S}$ is an abstract simplicial complex with vertex set $\{1,2, \ldots, n\}$. Let ${ }^{3}$

$$
\mathcal{K}(P, \mathcal{S})=\{\operatorname{conv}(P(\alpha)) \mid \alpha \in \mathcal{S}\} .
$$

$\mathcal{K}(P, \mathcal{S})$ is a geometric simplicial complex if

1. $\forall \alpha \in \mathcal{S}$, the points in $P(\alpha)$ are affinely independent

2. $s_{1}, s_{2} \in \mathcal{K}(P, \mathcal{S}) \Longrightarrow s_{1} \cap s_{2} \leq s_{1}, s_{2}$

and moreover, if these properties hold then $\operatorname{vert}(\mathcal{K}(P, \mathcal{S}))=P$. Proofs of these properties are provided in [20].

A triangulation ${ }^{4} \mathcal{T}$ is a geometric simplicial complex in $\mathbb{R}^{d}$ for which the underlying space is a $k$-manifold with boundary. Since a triangulation is a type of geometric simplicial complex, it can be parameterized in the same manner. We write $\mathcal{T}(P, \mathcal{S}):=\mathcal{K}(P, \mathcal{S})$ to indicate that the resulting geometric simplicial complex generated by the pair $(P, \mathcal{S})$ is a triangulation.

In this paper, we will only deal with triangulations which are $d$-manifolds with boundary that have a simply connected underlying space.

For notational convenience, we assume that the triangulation $\mathcal{T}=\mathcal{K}(P, \mathcal{S})$ has $N d$-simplices that have been indexed and named $S_{i}, i=1, \ldots, N$. Let $S_{i}, S_{j} \in \mathcal{T}$. We then define

$$
d_{i, j}:=\operatorname{dim}\left(S_{i} \cap S_{j}\right)=\operatorname{card}\left(\operatorname{vert}\left(S_{i} \cap S_{j}\right)\right)-1,
$$

the dimension of the face shared by $S_{i}$ and $S_{j}$. Let $N_{i}$ be the number of $d$-simplices in $\operatorname{St}\left\{p_{i}\right\}$,

$$
N_{i}=\sum_{S_{j} \in \operatorname{St} p_{i}} 1
$$

\subsection{Piecewise linear functions}

A continuous piecewise linear function $f_{\mathcal{P}}: D \subseteq \mathbb{R}^{d} \rightarrow \mathbb{R}^{d}$ is parameterized by a triplet $\mathcal{P}=(P, Q, \mathcal{S})$. $P$ is an indexed set of $n$ points in the domain and $Q$ is an indexed set of $n$ points in the codomain,

$$
P=\left\{p_{1}, \quad p_{2}, \ldots, \quad p_{n}\right\} \quad Q=\left\{\begin{array}{llll}
q_{1}, & q_{2}, \ldots, & q_{n}
\end{array}\right\} .
$$

$\mathcal{S}$ is an abstract simplicial complex of indices with $\operatorname{vert}(\mathcal{S})=\{1, \ldots, n\}$, such that $\mathcal{T}(P, \mathcal{S})$ is a triangulation and $|\mathcal{T}(P, \mathcal{S})|=D$. This defines a continuous piecewise linear function $f_{\mathcal{P}}$ such that $f_{\mathcal{P}}\left(p_{i}\right)=q_{i}$, and for

\footnotetext{
${ }^{2}$ This is not formally a parameterization, because there are some pairs $(P, \mathcal{S})$ for which $\mathcal{K}(P, \mathcal{S})$ is not a geometric simplicial complex. However, for any geometric simplicial complex $\mathcal{K}$, we can write down a pair $(P, \mathcal{S})$ such that $\mathcal{K}=\mathcal{K}(P, \mathcal{S})$.

${ }^{3}$ Formally, an indexed set $P$ of $n$ points in $\mathbb{R}^{d}$ is a map $P:\{1,2, \ldots, n\} \rightarrow \mathbb{R}^{d}$. The $i^{\text {th }}$ member of $P$ is $P(i)$, which we generally write as $p_{i}$ for notational convenience. Here we extend the notion of $P$ sets. Let $\alpha \subseteq\{1,2, \ldots, n\}$, then $P(\alpha):=\{P(i) \mid i \in \alpha\}$

${ }^{4}$ There is no formal definition of triangulation in geometry [13]. The definition of triangulation used here is slightly more general than used in [14], which requires that the underlying space be the convex hull of the vertex set. A triangulation as defined here which has a simply connected underlying space may be transformed to a triangulation as defined in [14] by a piecewise linear homeomorphism. The key concept in our definition is that a triangulation has good local volume properties everywhere. The underlying space has no "thin" spots. In topology, a triangulation of a topological space $\mathcal{X}$ is formally defined as a geometric simplicial complex $\mathcal{K}$ coupled with a homeomorphism between $|\mathcal{K}|$ and $\mathcal{X}$. The definition of triangulation used in this paper is more narrow than the topological notion.
} 
any $S \in \mathcal{T}(P, \mathcal{S}), f_{\mathcal{P}}(x)$ is affine on $S$. For a $d$-simplex $S_{i} \in \mathcal{T}(P, \mathcal{S})$ with $\operatorname{vert}\left(S_{i}\right)=\left\{p_{i_{1}}, p_{i_{2}}, \ldots, p_{i_{d+1}}\right\}$, the PL function $f_{\mathcal{P}}(x)$ for $x \in S_{i}$, is given by

$$
\left.f_{\mathcal{P}}\right|_{S_{i}}(x)=\left[\begin{array}{llll}
q_{i_{1}} & q_{i_{2}} & \cdots & q_{i_{d+1}}
\end{array}\right]\left[\begin{array}{cccc}
p_{i_{1}} & p_{i_{2}} & \cdots & p_{i_{d+1}} \\
1 & 1 & & 1
\end{array}\right]^{-1}\left[\begin{array}{l}
x \\
1
\end{array}\right] .
$$

Equation (2) uses a homogeneous representation for the rightmost two factors, though $\left.f_{\mathcal{P}}\right|_{S_{i}}$ can be equivalently expressed in the more typical form as $A_{i} x+b_{i}$. The $d$-simplices of $\mathcal{T}(P, \mathcal{S})$ are a cover for $D$. If $S_{i} \cap S_{j} \neq \emptyset$, and $\left.f_{\mathcal{P}}\right|_{S_{i}}(x)=A_{i} x+b_{i}$ and $\left.f_{\mathcal{P}}\right|_{S_{j}}(x)=A_{j} x+b_{j}$, then $A_{i} x+b_{i}=A_{j} x+b_{j}$ for $x \in S_{i} \cap S_{j}$. This follows from Claim A.6, which states that $\left(A_{i}-A_{j}\right)$ has a null space of dimension $d_{i, j}$ parallel to aff $\left(S_{i} \cap S_{j}\right)$.

One of the most compelling properties of piecewise linear functions is the ability to check invertibility and invert in closed form. Let $f_{\mathcal{P}}$ be a piecewise linear function parameterized by $\mathcal{P}=(P, Q, \mathcal{S})$. If $\mathcal{T}(Q, \mathcal{S})$ is a triangulation, then the piecewise linear function is invertible, and the inverse $f_{\mathcal{P}}{ }^{-1}$ is a piecewise linear function parameterized by $\mathcal{P}^{-1}=(Q, P, \mathcal{S})$. This is proven in Claim A.8.

Another important fact applied in proving the main result of Section 4 is the continuity of a PL function in its continuous parameters. This claim, stated formally in Claim A.7, establishes that two piecewise linear functions with the same combinatorial structure are close in the $L_{\infty}$ sense if their vertices are close in the Euclidean sense.

We call a piecewise linear function $f_{\mathcal{P}}$ parameterized by $\mathcal{P}=(P, Q, \mathcal{S})$ nondegenerate if for all $p_{i} \in P$ such that $p_{i} \notin \partial|\mathcal{T}(P, \mathcal{S})|$, the matrix $H_{i}$ is full rank, where

$$
H_{i}=\left(\frac{1}{N_{i}} \sum_{S_{j} \in \operatorname{St}\left\{p_{i}\right\}} A_{j}^{\mathrm{T}} A_{j}\right)-\overline{A^{i} \mathrm{~T}} \overline{A^{i}}, \quad \text { where } \overline{A^{i}}=\frac{1}{N_{i}} \sum_{S_{j} \in \operatorname{St}\left\{p_{i}\right\}} A_{j}
$$

Intuitively, nondegeneracy of $f_{\mathcal{P}}$ requires that for any $p_{i} \notin \partial|\mathcal{T}(P, \mathcal{S})|$ not all of the affine functions that $f_{\mathcal{P}}$ takes in the surrounding $d$-simplices are parallel.

\section{The minvar Algorithm}

The minvar algorithm is an iterative scheme to generate a locally good PL approximation to data. Similar to algorithms proposed in the possibly discontinuous piecewise polynomial approximation literature $[2,25$, $26,40]$, minvar takes advantage of the structure of piecewise linear functions. Let $\mathcal{Z}=\left\{\left(x_{i}, y_{i}\right)\right\}_{i=1}^{N_{s}}$, where $x_{i} \in D \subseteq \mathbb{R}^{d}$ and $y_{i} \in \mathbb{R}^{d}$, be the set of input-output data to be approximated. The minvar algorithm iteratively improves a piecewise linear approximation to the data, $f_{\mathcal{P}}^{(k)}$, parameterized by $\mathcal{P}^{(k)}=$ $\left(P^{(k)}, Q^{(k)}, \mathcal{S}\right)$, such that $\left|\mathcal{T}\left(P^{(k)}, \mathcal{S}\right)\right|=D$. (The superscript in parentheses indicates iteration number.) The algorithm breaks down into two stages. The first stage partitions the data according to the $d$-simplices of $\mathcal{T}\left(P^{(k)}, \mathcal{S}\right)$ and computes the least squares linear approximations for each subset of the data. This set of linear approximations is the optimal possibly discontinuous piecewise linear approximation on the partition $\mathcal{T}\left(P^{(k)}, \mathcal{S}\right)$. The second stage chooses $\left(P^{(k+1)}, Q^{(k+1)}\right)$ to make $f_{\mathcal{P}}^{(k+1)}$, a continuous PL function, be "close" to the discontinuous approximation from the first stage. The stages are then iterated.

Recall from the previous section that the domain of a piecewise linear function is $|\mathcal{T}(P, \mathcal{S})|$, so moving a vertex $p_{i} \in \partial|\mathcal{T}(P, \mathcal{S})|$, will change the domain of definition of the PL function. Since we desire a fixed domain for the PL function, the present exposition considers vertices on the domain boundary to be fixed. This can be relaxed to allow boundary vertices that are not extreme points to move in appropriately chosen affine subspaces using a constrained version of the cost function from step 3 of the minvar algorithm. Computational details of constrained motion will be presented in a subsequent paper on engineering applications of minvar.

From an initial parameterization $\mathcal{P}^{(0)}=\left(P^{(0)}, Q^{(0)}, \mathcal{S}\right)$, the minvar algorithm generates a sequence of parameterizations, $\mathcal{P}^{(k)}=\left(P^{(k)}, Q^{(k)}, \mathcal{S}\right)$, as follows: 
1. Partition the data set $\mathcal{Z}$ into subsets $\mathcal{Z}_{j}$ corresponding to the $d$-simplices, $S_{1}, \ldots, S_{N}$ of $\mathcal{T}\left(P^{(k)}, \mathcal{S}\right)$, breaking multiple memberships (data points that lie on the boundary between $d$-simplices) systematically

2. Compute the least squares affine approximation, $L_{j}(x)$, for each subset $\mathcal{Z}_{j}$

3. Update the vertex locations,

$$
\begin{aligned}
p_{i}^{(k+1)} & =\underset{x \in \mathbb{R}^{d}}{\arg \min } \operatorname{var} L^{i}(x)+\lambda\left\|x-p_{i}^{(k)}\right\|^{2} & & \forall p_{i}^{(k)} \notin \partial\left|\mathcal{T}\left(P^{(k)}, \mathcal{S}\right)\right| \\
p_{i}^{(k+1)} & =p_{i}^{(k)} & & \text { otherwise } \\
q_{i}^{(k+1)} & =\frac{1}{N_{i}} \sum_{S_{j} \in \operatorname{St}\left\{p_{i}\right\}} L^{i}\left(p_{i}^{(k+1)}\right) & & \forall i
\end{aligned}
$$

where $L_{j}(x)=A_{j} x+b_{j}$ and

$$
\operatorname{var} L^{i}(x)=\sum_{S_{j} \in \operatorname{St}\left\{p_{i}\right\}}\left(L_{j}(x)-\frac{1}{N_{i}} \sum_{S_{k} \in \operatorname{St}\left\{p_{i}\right\}} L_{k}(x)\right)^{2}
$$

4. If the vertices are not converged, then $k \leftarrow k+1$, go to 1

Notice that (3) is positive definite quadratic function of $x$, and thus can be minimized in closed form by "completing the square," with the solution given by

$$
p_{i}^{(k+1)}=-H_{i}^{-1} h_{i}
$$

where

$$
\begin{array}{r}
H_{i}=\left[\frac{1}{N_{i}} \sum_{S_{j} \in \operatorname{St}\left\{p_{i}\right\}} A_{j}^{\mathrm{T}} A_{j}\right]-\overline{A^{i} \mathrm{~T}} \overline{A^{i}}+\lambda I \\
h_{i}=\left[\frac{1}{N_{i}} \sum_{S_{j} \in \operatorname{St}\left\{p_{i}\right\}} A_{j}{ }^{\mathrm{T}} b_{j}\right]-\overline{A^{i} \mathrm{~T}} \overline{b^{i}}-\lambda p_{i}^{(k)} \\
\overline{A^{i}}=\frac{1}{N_{i}} \sum_{S_{j} \in \operatorname{St}\left\{p_{i}\right\}} A_{j} \quad \overline{b^{i}}=\frac{1}{N_{i}} \sum_{S_{j} \in \operatorname{St}\left\{p_{i}\right\}} b_{j}
\end{array}
$$

The non-negative quantity var $L^{i}(x)$ measures, as a function of location in the domain $x$, how tightly clustered the range values generated from the least squares approximations on $d$-simplices in $\operatorname{St}\left\{p_{i}\right\}$ are. In the case of a scalar domain, an interior vertex $p_{i}$ is in at most two 1-simplices. Thus, if the least squares approximations are not parallel, then $\operatorname{var} L^{i}\left(x_{c}\right)=0$ at and only at $x_{c}$, the domain value of the point at which the least squares approximations intersect. In the scalar case, the minvar algorithm with $\lambda=0$ moves the domain and codomain vertices to the intersection point of the least squares approximations. We called our initial scalar algorithm the "Graph Intersection" algorithm [22] due to this fact. For dimensions higher than 1, there is generically no unique intersection point for the least squares approximations surrounding $p_{i}$, due to the geometry of triangulations. Rather than the intersection point, minvar with $\lambda=0$ picks the point where the range values are most tightly clustered.

The $\lambda$ term in (3) is a regularization. It guarantees that (3) will have a unique minimum, even if all the least squared approximations are parallel. More importantly, in the implementation of minvar the $\lambda$ 
parameter can be tuned to prevent a vertex from jumping long distances and creating a "tangle" in the domain triangulation of the approximation. A tangle is when movement of the vertices causes $\mathcal{T}(P, \mathcal{S})$ to no longer be a geometric simplicial complex. That is, either a simplex has been "flattened" or there are simplices whose intersection is not another simplex from the complex. This generally occurs when a domain vertex moves through one of its opposing faces. Methods for detecting and correcting triangulation tangles will be covered in a subsequent paper on using minvar in engineering applications.

In this exposition, minvar does not modify the combinatorial structure, $\mathcal{S}$, of the PL approximation. Heuristics for adapting the domain triangulation of a 2-dimensional PL function are presented in [12, 11] for interpolation and [40] for approximation. These heuristics flip edges in the domain triangulation to improve a local goodness criterion, similar to a method for computing the planar Delaunay triangulation. Generalizing these heuristics to higher dimensions is difficult because local topological changes of the triangulation in dimensions greater than two are more complex than edge flipping [28, 14]. Nonetheless, we find that adaptation of the combinatorial parameters of the PL function via topological flipping provides significant benefit in practice. Techniques for adapting the combinatorial structure will be presented in a subsequent paper on engineering applications of minvar.

\section{A Local Convergence Proof for the minvar Algorithm}

We turn now to the central result: a local convergence proof for the minvar algorithm. The result is for the "approximation," as opposed to "estimation," version of the minvar algorithm. That is, the data generating function is considered to be directly available in closed form, rather than through a set of discrete data. In this case, the least squares approximations from Step 2 become $L_{2}$ orthogonal projections of $\left.f_{\mathcal{P}}^{*}\right|_{S_{i}}$, the data generating function restricted to $S_{i}$, to the space of affine functions. Since the data or data generating function only appear in Step 2, the approximation version may be viewed as the limit behavior of the estimation version when provided with an unbounded quantity of uniformly distributed data.

Theorem 1 shows that, if the data generating function is a nondegenerate piecewise linear function and the approximation is initialized "close enough" to the data generating function, then the minvar algorithm with $\lambda=0$ will cause the approximation to converge to the data generating function in the $L_{\infty}$ sense. In this case, "close enough" means that the initial approximation shares the same combinatorial structure as the data generating function, and the vertices of the approximation start close to the corresponding vertices of the data generating function. Examining minvar when $\lambda=0$ admits a simpler proof while capturing the essence of the algorithm. Similar results could be obtained for $\lambda>0$, though the convergence rate would be slower. An additional technical condition, that the data generating function be nondegenerate, is required when $\lambda=0$ in order to guarantee existence of a unique solution to (3), whereas for $\lambda>0$ the regularized variance minimization in (3) is guaranteed to have a unique solution.

In this paper, unless otherwise noted, vector norms are the standard Euclidean norm and matrix norms are the induced two norm.

Theorem 1. Let $f_{\mathcal{P}}^{*}$ be a nondegenerate piecewise linear data generating function parameterized by $\mathcal{P}^{*}=$ $\left(P^{*}, Q^{*}, \mathcal{S}^{*}\right)$. Let $\epsilon_{0}=\epsilon_{0}\left(\mathcal{P}^{*}\right)$ be given by (13). Let the initial approximation $f_{\mathcal{P}}^{(0)}$ be parameterized by $\left(P^{(0)}, Q^{(0)}, \mathcal{S}^{*}\right)$, satisfying for some $\epsilon<\epsilon_{0},\left\|p_{i}^{(0)}-p_{i}^{*}\right\|<\epsilon$ for all $i$. Then application of the minvar algorithm with $\lambda=0$ yields a sequence of approximations satisfying

$$
\lim _{j \rightarrow \infty}\left\|f_{\mathcal{P}}^{(j)}-f_{\mathcal{P}}^{*}\right\|_{\infty}=0
$$

Proof. Proposition 2 shows that iteration of the minvar algorithm causes the vertices of the approximation to converge to the vertices of the data generating function. By Claim A.7, a PL function is continuous in its vertices. The theorem follows directly.

The theorem follows readily from Proposition 2, which likewise follows readily from Proposition 1 . The statements and proofs of the propositions and lemmas follow in the next subsections, but first we offer a 
short sketch of the structure of the proof. The essence of Proposition 1 is that when the distances between the vertices of the approximation and the corresponding vertices of the data generating function are bounded by $\epsilon$, then after one iteration of the minvar algorithm the distances will be bounded by a constant times $\epsilon^{2}$. This result is established by applying two lemmas corresponding to the two stages of the algorithm. Lemma 1 proves that if the distances between corresponding vertices are bounded by $\epsilon$, then the perturbation of the least squares affine map over a given simplex of the approximation from the affine map in the corresponding simplex of the data generating function is bounded by a constant times $\epsilon^{2}$. Lemma 2 proves that if the perturbation of the least squares affine map over a simplex of the approximation from the affine map in the corresponding simplex of the data generating function is bounded by $\Delta$, then the variance minimization will place the new vertices of the approximation such that the distance between them and the corresponding vertices of the data generating function are bounded by a constant times $\Delta$. The combination of Lemma 1 and 2 provides Proposition 1.

The quadratic rate of convergence in Proposition 1 arises from the hypothesis that the data generating function is piecewise linear and close to the initial approximation. Without this assumption, Lemma 1 would fail to provide an $\epsilon^{2}$ perturbation in the least squares affine approximations. In this case, we suspect the convergence rate of the algorithm to be linear. Convergence may be slower on fine triangulations, but since this algorithm is intended primarily for use with a discrete set of data, the fineness of the triangulation is inherently limited by the amount of data provided. In applications, minvar can run triangulations of practical size in a few minutes.

\subsection{Lemmas and Propositions}

This section states the lemmas and propositions, while the proofs are provided in the following section. First, we introduce several reoccurring constants. These constants may be interpreted geometrically as minima or maxima of different measures of the "radii" of $d$-simplices in the triangulation $\mathcal{T}\left(P^{*}, \mathcal{S}^{*}\right)$ of the data generating function. The first measures the maximum inter-vertex distance between "connected" vertices,

$$
r_{1}:=\max _{\substack{S^{*} \in \mathcal{T}\left(P^{*}, \mathcal{S}^{*}\right) \\ p_{i}^{*}, p_{j}^{*} \in S^{*}}}\left\|p_{i}^{*}-p_{j}^{*}\right\|
$$

The second measures the minimum distance of a vertex to its opposing hyperplanes,

$$
r_{2}:=\min _{\substack{S^{*} \in \mathcal{T}\left(P^{*}, \mathcal{S}^{*}\right) \\ p^{*} \in \operatorname{vert}\left(S^{*}\right)}} \delta\left(p^{*}, \operatorname{aff}\left(\operatorname{vert}\left(S^{*}\right)-\left\{p^{*}\right\}\right)\right) .
$$

The third measures the $d$-simplex which can be dilated the least before it intersects simplices outside its immediate neighborhood.

$$
r_{3}:=\min _{S^{*} \in \mathcal{T}\left(P^{*}, \mathcal{S}^{*}\right)} \sup \left\{\epsilon\left|\mathcal{D}\left(S^{*}, \epsilon\right) \subseteq\right| \mathrm{ClSt} S^{*} \mid\right\}
$$

The first lemma shows that if the domain vertices of the approximation are close to the domain vertices of the data generating function, then least squares affine fit in a simplex $S_{i}$ is a perturbation away from the affine function that the data generating function takes in $S_{i}^{*}$. Moreover, the perturbation is quadratic in the bound on the distance between the approximation and data generating function's domain vertices. We write $\Pi(f)$ to denote the $L_{2}$ orthogonal projection of the function $f$ onto the space of affine functions.

Lemma 1. Let $f_{\mathcal{P}}^{*}$ be a piecewise linear data generating function parameterized by $\mathcal{P}^{*}=\left(P^{*}, Q^{*}, \mathcal{S}^{*}\right)$. Consider a piecewise linear approximation $f_{\mathcal{P}}$ parameterized by $\mathcal{P}=\left(P, Q, \mathcal{S}^{*}\right)$. Let $\epsilon<\epsilon_{c}$, where

$$
\epsilon_{c}:=\min \left\{\frac{1}{2(d+1)} r_{2}, \quad r_{3}, \quad 1\right\} .
$$

Consider the simplices $S_{i}^{*}$ and $S_{i}$. Let $x_{c} \in S_{i}^{*}$. Let $\left.f_{\mathcal{P}}^{*}\right|_{S_{i}^{*}}(x)=A_{i}^{*}\left(x-x_{c}\right)+b_{i}^{*}$. If $\left\|p_{j}-p_{j}^{*}\right\|<\epsilon$ for

all $p_{j}^{*} \in S_{i}^{*}$, then the least squares approximation to $f_{\mathcal{P}}^{*}$ on $S_{i}, \Pi\left(\left.f_{\mathcal{P}}^{*}\right|_{S_{i}}\right)(x)=\hat{A}_{i}\left(x-x_{c}\right)+\hat{b}_{i}$, satisfies the 
property

$$
\left\|\left[\begin{array}{c}
\hat{A}_{i}^{\mathrm{T}}-A_{i}^{* \mathrm{~T}} \\
\hat{b}_{i}^{\mathrm{T}}-b_{i}^{* \mathrm{~T}}
\end{array}\right]\right\|_{2}<c_{1, i} \epsilon^{2},
$$

where $c_{1, i}=c_{1, i}\left(\mathcal{P}^{*}\right)$ is given by (24).

The second lemma considers one set of affine functions that all intersect at a common point and another set of affine functions which are perturbations of the first set of functions. It is shown the performing the variance minimization, equivalent to (3) with $\lambda=0$, on the second set of functions generates a point whose distance from the intersection point is linear in the norm of the perturbations.

Lemma 2. Let $L^{*}$ be a set of $N$ affine maps, $L_{1}^{*}, \ldots L_{N}^{*}$, such that all intersect at $\left(p^{*}, q^{*}\right)$ and are written as $L_{i}^{*}(x)=A_{i}^{*}\left(x-p^{*}\right)+q^{*}$, and such that $H^{*}$, given by (26), is full rank. Let $L$ be a set of perturbed affine maps, $L_{1}, \ldots L_{N}$, expressed as $L_{i}(x)=\hat{A}_{i}\left(x-p^{*}\right)+\hat{q}_{i}$, which satisfy the property

$$
\left\|\left[\begin{array}{c}
\hat{A}_{i}^{\mathrm{T}}-A_{i}^{* \mathrm{~T}} \\
\hat{q}_{i}^{\mathrm{T}}-q^{* \mathrm{~T}}
\end{array}\right]\right\|<\Delta
$$

for $\Delta<\Delta_{0}$, where $\Delta_{0}=\Delta_{0}\left(A_{i}^{*}, p^{*}, q^{*}\right)$ is given by (25). Let $p^{\prime}$ and $q^{\prime}$ be given by

$$
\begin{aligned}
& p^{\prime}=\underset{x}{\arg \min } \quad \operatorname{var} L(x) \\
& q^{\prime}=\frac{1}{N} \sum_{i=1}^{N} L\left(p^{\prime}\right) .
\end{aligned}
$$

Then $p^{\prime}$ and $q^{\prime}$ satisfy

$$
\begin{aligned}
& \left\|p^{\prime}-p^{*}\right\|<c_{2} \Delta \\
& \left\|q^{\prime}-q^{*}\right\|<c_{3} \Delta
\end{aligned}
$$

where $c_{2}=c_{2}\left(A_{i}^{*}, p^{*}, q^{*}\right)$ and $c_{3}=c_{3}\left(A_{i}^{*}, p^{*}, q^{*}\right)$ are given by (29) and (30).

The first proposition brings the two lemmas together to show that a single step of the minvar algorithm induces a quadratic change in the distance of the approximation vertices to the data generating function vertices.

Proposition 1. Let $f_{\mathcal{P}}^{*}$ be a nondegenerate piecewise linear data generating function parameterized by $\mathcal{P}^{*}=$ $\left(P^{*}, Q^{*}, \mathcal{S}^{*}\right)$. Let $\epsilon<\epsilon_{d}$,

$$
\epsilon_{d}:=\min \left\{\epsilon_{c}, \sqrt{\frac{\Delta_{0}^{m}}{c_{1}}}\right\},
$$

where $\epsilon_{c}=\epsilon_{c}\left(\mathcal{P}^{*}\right)$ is given by (10), $\Delta_{0}^{m}=\Delta_{0}^{m}\left(\mathcal{P}^{*}\right)$ by (31), and $c_{1}=c_{1}\left(\mathcal{P}^{*}\right)$ by (33).

If the piecewise linear approximation $f_{\mathcal{P}}$ parameterized by $\left(P, Q, \mathcal{S}^{*}\right)$ satisfies $\left\|p_{i}-p_{i}^{*}\right\|<\epsilon$ for all $i$, then one iteration of the minvar algorithm with $\lambda=0$ gives the new approximation $f_{P}^{\prime}$ parameterized by $\left(P^{\prime}, Q^{\prime}, \mathcal{S}^{*}\right)$, which satisfies

$$
\begin{aligned}
\left\|p_{i}^{\prime}-p_{i}^{*}\right\| & <c_{4} \epsilon^{2} \\
\left\|q_{i}^{\prime}-q_{i}^{*}\right\| & <c_{5} \epsilon^{2}
\end{aligned}
$$

for all $i$, where $c_{4}=c_{4}\left(\mathcal{P}^{*}\right)$ and $c_{5}=c_{5}\left(\mathcal{P}^{*}\right)$ are given by (37) and (38).

The second proposition applies the first proposition to show that iteration of the minvar algorithm cause convergence of the vertices of the approximation to the vertices of the data generating function. 
Proposition 2. Let $f_{\mathcal{P}}^{*}$ be a nondegenerate piecewise linear data generating function parameterized by $\left(P^{*}, Q^{*}, \mathcal{S}^{*}\right)$. Let

$$
\epsilon_{0}=\min \left\{\epsilon_{d}, \frac{1}{c_{4}}\right\}
$$

where $\epsilon_{d}$ and $c_{4}$ are given in Proposition 1. If for some $0<\epsilon<\epsilon_{0}$ the initial piecewise linear approximation $f_{P}^{(0)}$ with parameterization $\left(P^{(0)}, Q^{(0)}, \mathcal{S}^{*}\right)$ satisfies $\left\|p_{i}^{(0)}-p_{i}^{*}\right\|<\epsilon$ for all $i$, then iteration of the minvar algorithm with $\lambda=0$ gives a sequence of approximations $f_{P}^{(k)}$ satisfying

$$
\begin{aligned}
\lim _{k \rightarrow \infty}\left\|p_{i}^{(k)}-p_{i}^{*}\right\| & =0 \\
\lim _{k \rightarrow \infty}\left\|q_{i}^{(k)}-q_{i}^{*}\right\| & =0
\end{aligned}
$$

for all $i$.

\subsection{Proofs of Lemmas and Propositions}

This section presents proofs of the lemmas and propositions stated in the previous section.

Lemma 1. Let $\varphi_{i}(x):=A_{i}^{*}\left(x-x_{c}\right)+b_{i}^{*}$, the extension of $\left.f_{\mathcal{P}}^{*}\right|_{S_{i}^{*}}$ to the entire domain. Let $\psi_{i}(x):=f_{\mathcal{P}}^{*}(x)-$ $\varphi_{i}(x)$.

The orthogonal projection $\Pi$ is a linear operator, and moreover for $g$ affine, $\Pi(g)=g$. It follows that

$$
\begin{aligned}
\Pi\left(\left.f_{\mathcal{P}}^{*}\right|_{S_{i}}\right) & =\Pi\left(\left.\varphi_{i}\right|_{S_{i}}\right)+\Pi\left(\left.\psi_{i}\right|_{S_{i}}\right) \\
& =\varphi_{i}+\Pi\left(\left.\psi_{i}\right|_{S_{i}}\right) .
\end{aligned}
$$

Let $\hat{A}_{i}$ and $\hat{b}_{i}$ be such that $\Pi\left(\left.f_{\mathcal{P}}^{*}\right|_{S_{i}}\right)(x)=\hat{A}_{i}\left(x-x_{c}\right)+\hat{b}_{i}$. Then from (14) it follows that $\Pi\left(\left.\psi_{i}\right|_{S_{i}}\right)=$ $\left(\hat{A}_{i}-A_{i}^{*}\right)\left(x-x_{c}\right)+\left(\hat{b}_{i}-b_{i}^{*}\right)$. Moreover, since $\Pi\left(\left.\psi_{i}\right|_{S_{i}}\right)=\Pi\left(\left.f_{\mathcal{P}}^{*}\right|_{S_{i}}-\left.\varphi_{i}\right|_{S_{i}}\right)$, we can compute $\left(\hat{A}_{i}-A_{i}^{*}\right)$ and $\left(\hat{b}_{i}-b_{i}^{*}\right)$ using the formula for $L_{2}$ orthogonal projection of $\left.f_{\mathcal{P}}^{*}\right|_{S_{i}}-\left.\varphi_{i}\right|_{S_{i}}$,

$$
\begin{gathered}
{\left[\begin{array}{c}
\left(\hat{A}_{i}-A_{i}^{*}\right)^{\mathrm{T}} \\
\left(\hat{b}_{i}-b_{i}^{*}\right)^{\mathrm{T}}
\end{array}\right]=S_{x x, i}{ }^{-1} S_{x y, i},} \\
S_{x x, i}=\int_{S_{i}}\left[\begin{array}{c}
x-x_{c} \\
1
\end{array}\right]\left[\begin{array}{cc}
x^{\mathrm{T}}-x_{c}{ }^{\mathrm{T}} & 1
\end{array}\right] d x, \quad S_{x y, i}=\int_{S_{i}}\left[\begin{array}{c}
x-x_{c} \\
1
\end{array}\right]\left(f_{\mathcal{P}}^{*}(x)-\varphi_{i}(x)\right)^{\mathrm{T}} d x .
\end{gathered}
$$

The submultiplicative property holds for the induced two norm,

$$
\left\|\left[\begin{array}{c}
\left(\hat{A}_{i}-A_{i}^{*}\right)^{\mathrm{T}} \\
\left(\hat{b}_{i}-b_{i}^{*}\right)^{\mathrm{T}}
\end{array}\right]\right\| \leq\left\|S_{x x, i}{ }^{-1}\right\|\left\|S_{x y, i}\right\|,
$$

so we can independently establish bounds on $\left\|S_{x x, i}{ }^{-1}\right\|$ and $\left\|S_{x y, i}\right\|$. We will proceed to bound $\left\|S_{x x, i}{ }^{-1}\right\|$. Since $S_{i}$ is a $d$-simplex, it follows from calculus and the definition of $S_{x x, i}$ that $S_{x x, i}$ is a positive definite matrix. Let $M_{i}^{*}$ be given by

$$
M_{i}^{*}:=\int_{\mathcal{D}\left(S_{i}^{*},-\epsilon_{c}\right)}\left[\begin{array}{c}
x-x_{c} \\
1
\end{array}\right]\left[x^{\mathrm{T}}-x_{c}^{\mathrm{T}} \quad 1\right] d x
$$

Since $0<\epsilon<\epsilon_{c}$ by hypothesis and $\epsilon_{c} \leq \frac{1}{2(d+1)} r_{2}$ by definition, it follows from Claim A.3 that $\mathcal{D}\left(S_{i}^{*},-\epsilon_{c}\right)$ is a $d$-simplex. Thus $M_{i}^{*}$ is also positive definite, and hence invertible. Since $\epsilon<\epsilon_{c}$ and the vertices or $S_{i}$ are all less than $\epsilon$ away from the vertices of $S_{i}^{*}$, it follows that $\mathcal{D}\left(S_{i}^{*},-\epsilon_{c}\right) \subseteq S_{i}$. Thus, $x^{\mathrm{T}} M_{i}^{*} x<x^{\mathrm{T}} S_{x x, i} x$ for all $x$, which implies that $\lambda_{\min }(M)<\lambda_{\min }\left(S_{x x, i}\right)$. This provides the bound on $\left\|S_{x x, i}{ }^{-1}\right\|$,

$$
\left\|S_{x x, i}{ }^{-1}\right\|<\left\|M_{i}^{*-1}\right\| .
$$


Now we proceed to $\left\|S_{x y, i}\right\|$. By the properties of norms,

$$
\left\|S_{x y, i}\right\| \leq \int_{S_{i}}\left\|\left[\begin{array}{c}
x-x_{c} \\
1
\end{array}\right]\right\|\left\|\psi_{i}(x)\right\| d x
$$

Let $\mathcal{C}\left(S_{i}, \epsilon\right)=\left\{x \in \mathbb{R}^{d} \mid \delta\left(x, S_{i}\right) \leq \epsilon\right\}$. By hypothesis, the vertices of $S_{i}$ are less than $\epsilon$ away from the vertices of $S_{i}^{*}$, so vert $S_{i} \subseteq \mathcal{C}\left(S_{i}^{*}, \epsilon\right)$. Moreover, since both $S_{i}$ and $\mathcal{C}\left(S_{i}^{*}, \epsilon\right)$ are convex, $S_{i} \subseteq \mathcal{D}\left(S_{i}^{*}, \epsilon\right)$. The integrand in (15) is nonnegative definite, so (15) is bounded by

$$
\leq \int_{\mathcal{C}\left(S_{i}^{*}, \epsilon\right)}\left\|\left[\begin{array}{c}
x-x_{c} \\
1
\end{array}\right]\right\|\left\|\left(\psi_{i}(x)\right)\right\| d x
$$

By hypothesis $x_{c} \in S_{i}^{*}$. By the definition of $r_{1}$ and since $\epsilon<\epsilon_{c}$, it follows that $\forall x \in \mathcal{C}\left(S_{i}^{*}, \epsilon\right),\left\|x-x_{c}\right\| \leq$ $\bar{r}:=r_{1}+2 \epsilon_{c}$. Thus, the integral above is further bounded by

$$
\leq \sqrt{1+\bar{r}^{2}} \int_{\mathcal{C}\left(S_{i}^{*}, \epsilon\right)}\left\|\left(\psi_{i}(x)\right)\right\| d x
$$

Once again the integrand is nonnegative definite, so (16) can be bounded by integrating over $\mathcal{D}\left(S_{i}^{*}, \epsilon\right)$, since $\mathcal{C}\left(S_{i}^{*}, \epsilon\right) \subseteq \mathcal{D}\left(S_{i}^{*}, \epsilon\right)$,

$$
\begin{aligned}
& \leq \quad \sqrt{1+\bar{r}^{2}} \int_{\mathcal{D}\left(S_{i}^{*}, \epsilon\right)}\left\|\left(\psi_{i}(x)\right)\right\| d x \\
& =\sqrt{1+\bar{r}^{2}} \sum_{j=1}^{N} \int_{U_{j}}\left\|\left(\psi_{i}(x)\right)\right\| d x
\end{aligned}
$$

where $U_{j}=\mathcal{D}\left(S_{i}^{*}, \epsilon\right) \cap S_{j}^{*}$ and $N$ is the total number of $d$-simplices in the domain triangulation. Since $\psi_{i}(x)=0$ on $S_{i}^{*}$, the term corresponding to $j=i$ in (18) is 0 . By hypothesis $\epsilon<\epsilon_{c} \leq r_{3}$, so then following from the definition of $r_{3}, S_{j}^{*} \cap \mathcal{D}\left(S_{i}^{*}, \epsilon\right) \neq \emptyset$ if and only if $S_{j}^{*} \in \operatorname{St} S_{i}^{*}$. Thus the terms in (18) are only nonzero for $j$ such that $S_{j}^{*}$ is incident to $S_{i}^{*}$. Consider such a term,

$$
\int_{U_{j}}\left\|\psi_{i}(x)\right\| d x=\int_{U_{j}}\left\|\left(A_{j}^{*}-A_{i}^{*}\right)\left(x-x_{c}\right)+b_{j}^{*}-b_{i}^{*}\right\| d x,
$$

where $\left.f_{\mathcal{P}}^{*}\right|_{S_{j}^{*}}(x)=A_{j}^{*}\left(x-x_{c}\right)+b_{j}^{*}$. By Claim A.6, there exists $x_{O} \in S_{j}^{*} \cap S_{i}^{*} \subseteq U_{j}$ such that $\left(A_{j}^{*}-A_{i}^{*}\right)\left(x_{O}-\right.$ $\left.x_{c}\right)+b_{j}^{*}-b_{i}^{*}=0$. Applying the change of coordinates $y=x-x_{O}$ gives

$$
\int_{U_{j}}\left\|\psi_{i}(x)\right\| d x=\int_{U_{j}-x_{O}}\left\|\left(A_{j}^{*}-A_{i}^{*}\right) y\right\| d y
$$

Let $L$ be the linear subspace parallel to $\operatorname{aff}\left(S_{i}^{*} \cap S_{j}^{*}\right)$. Recall that $\operatorname{dim} L=\operatorname{dim} S_{i}^{*} \cap S_{j}^{*}:=d_{i, j}$. By Claim A.6, $L \subseteq \mathcal{N}\left(\left(A_{j}^{*}-A_{i}^{*}\right)\right)$. Let $v_{1}, \ldots, v_{d_{i, j}}$ be an orthonormal basis for $L$. Let $v_{d_{i, j}+1}, \ldots, v_{d}$ be an orthonormal basis for $L^{\perp}$. Then $P=\left[\begin{array}{llll}v_{1} & v_{2} & \cdots & v_{d}\end{array}\right]$ is an orthogonal matrix. Rewrite (19) under the change of coordinates $z=P^{\mathrm{T}} y$,

$$
=\int_{P^{\mathrm{T}}\left(U_{j}-x_{O}\right)}\left\|\left(A_{j}^{*}-A_{i}^{*}\right) P z\right\| d z
$$

Since the integrand is a nonnegative definite function, we may bound (20) by increasing the volume over which the integrand is integrated. By Claim A.4, there exists $\kappa_{i, j}$ such that for all $x \in U_{j}, \delta\left(x, \operatorname{aff}\left(S_{i}^{*} \cap S_{j}^{*}\right)\right)<\kappa_{i, j} \epsilon$. Equivalently $\delta(y, L)<\kappa_{i, j} \epsilon$ for any $y \in U_{j}-x_{O}$, from which it follows that the projection of $y$ onto $L^{\perp}$ must have magnitude less than $\kappa_{i, j} \epsilon$. Moreover, by the definition of $\bar{r}$, the projection of $y$ onto $L$ must have magnitude less than $\bar{r}$. It follows that $P^{\mathrm{T}}\left(U_{j}-x_{O}\right) \subseteq[-\bar{r}, \bar{r}]^{d_{i, j}} \times B_{\bar{d}_{i, j}}\left(\kappa_{i, j} \epsilon\right)$, where $\bar{d}_{i, j}=d-d_{i, j}$ and $B_{\bar{d}_{i, j}}\left(\kappa_{i, j} \epsilon\right)$ is the $\bar{d}_{i, j}$-dimensional ball of radius $\kappa_{i, j} \epsilon$. Then $(20)$ is bounded by

$$
\leq \int_{[-\bar{r}, \bar{r}]^{d_{i, j}} \times B_{\bar{d}_{i, j}}\left(\kappa_{i, j} \epsilon\right)}\left\|\left(A_{j}^{*}-A_{i}^{*}\right) P z\right\| d z
$$


The first $d_{i, j}$ columns of $\left(A_{j}^{*}-A_{i}^{*}\right) P$ are zero, since the first $d_{i, j}$ columns of $P$ are in the nullspace of $\left(A_{j}^{*}-A_{i}^{*}\right)$. Thus, the integrand in (21) has no dependence on $z_{1}, z_{2}, \ldots, z_{d_{i, j}}$, so we can integrate through for $z_{1}, \ldots, z_{d_{i, j}}$, giving

$$
=(2 \bar{r})^{d_{i, j}} \int_{B_{\bar{d}_{i, j}}\left(\kappa_{i, j} \epsilon\right)}\left\|\left(A_{j}^{*}-A_{i}^{*}\right) P\left[\begin{array}{l}
I \\
0
\end{array}\right] \bar{z}_{1}\right\| d z_{d_{i, i}+1} \ldots d z_{d}
$$

where $\bar{z}_{1}^{\mathrm{T}}=\left[\begin{array}{lll}z_{d_{i, j}+1} & \cdots & z_{d}\end{array}\right]^{\mathrm{T}}$. Since the first $d_{i, j}$ columns of $\left(A_{j}^{*}-A_{i}^{*}\right) P$ are zero and $P$ is orthogonal, it follows that $\left\|\left(A_{j}^{*}-A_{i}^{*}\right) P\left[\begin{array}{ll}I & 0\end{array}\right]^{\mathrm{T}}\right\| \leq\left\|A_{j}^{*}-A_{i}^{*}\right\|$. Thus, (22) can be further bound by

$$
\leq(2 \bar{r})^{d_{i, j}}\left\|A_{j}^{*}-A_{i}^{*}\right\| \int_{B_{\bar{d}_{i, j}}\left(\kappa_{i, j} \epsilon\right)}\left\|\bar{z}_{1}\right\| d \bar{z}_{1}
$$

From calculus (see for example [38]) it can be shown that ${ }^{5}$

$$
\int_{B_{k}(\epsilon)}\|w\| d w=\frac{k}{k+1} \frac{\pi^{k / 2}}{\Gamma(k / 2)} \epsilon^{k+1}
$$

Applying this with (23) to (18), and then simplifying using the fact that $\epsilon^{m} \leq \epsilon^{2}$ for $m \geq 2$ since $\epsilon<\epsilon_{c} \leq 1$, gives

$$
\left\|S_{x y, i}\right\| \leq \sqrt{1+\bar{r}^{2}} l_{i} \max _{\substack{j, \mathrm{~s} . t . j \neq i, j \\ S_{j}^{S *} \in \mathrm{St}_{i}^{*}}}\left((2 \bar{r})^{d_{i, j}^{*}}\left\|A_{j}^{*}-A_{i}^{*}\right\| \kappa_{i, j}{ }^{1+\bar{d}_{i, j}} \frac{\bar{d}_{i, j}}{\bar{d}_{i, j}+1} \frac{\pi^{\left(\bar{d}_{i, j}\right) / 2}}{\Gamma\left(\left(\bar{d}_{i, j}\right) / 2\right)}\right) \epsilon^{2}
$$

where $l_{i}=\sum_{S_{j}^{*} \in \operatorname{St} S_{i}^{*}} 1$. Then

$$
\begin{aligned}
\left\|\left[\begin{array}{c}
\hat{A}_{i}^{\mathrm{T}}-A_{i}^{* \mathrm{~T}} \\
\hat{b}_{i}^{\mathrm{T}}-b_{i}^{* \mathrm{~T}}
\end{array}\right]\right\| & \leq\left\|S_{x x, i}{ }^{-1}\right\|\left\|S_{x y, i}\right\| \\
& <c_{1, i} \epsilon^{2}
\end{aligned}
$$

where

$$
c_{1, i}:=\left\|M_{i}^{*-1}\right\| \sqrt{1+\bar{r}^{2}} l_{\substack { i \\
\begin{subarray}{c}{s . t . \\
\text { s.t. } \\
S_{j}^{*} \in \mathrm{St}_{i}^{*}{ i \\
\begin{subarray} { c } { s . t . \\
\text { s.t. } \\
S _ { j } ^ { * } \in \mathrm { St } _ { i } ^ { * } } }\end{subarray}}\left((2 \bar{r})^{d_{i, j}}\left\|A_{j}^{*}-A_{i}^{*}\right\| \kappa_{i, j}{ }^{1+\bar{d}_{i, j}} \frac{\bar{d}_{i, j}}{\bar{d}_{i, j}+1} \frac{\pi^{\left(\bar{d}_{i, j}\right) / 2}}{\Gamma\left(\left(\bar{d}_{i, j}\right) / 2\right)}\right)
$$

and $\bar{d}_{i, j}=d-d_{i, j}, l_{i}=\sum_{S_{j}^{*} \in \operatorname{St} S_{i}^{*}} 1$, and $\bar{r}=r_{1}+2 \epsilon_{c}$.

Lemma 2. Let the constant $\Delta_{0}$ from the statement of the lemma be given by

$$
\Delta_{0}:=\min \left\{\frac{1}{N} \sum_{j=1}^{N}\left\|A_{j}^{*}\right\|, \quad\left(\frac{12}{N}\left\|H^{*-1}\right\| \sum_{j=1}^{N}\left\|A_{j}^{*}\right\|\right)^{-1}\right\},
$$

where $H^{*}$ is given by $(26)$.

Solving $p^{\prime}=\arg \min _{x}$ var $L(x)$ is equivalent to solving (3) with $\lambda=0$. As with (3), a closed form expression for $p^{\prime}$ can be found by "completing the square." Specifically, $p^{\prime}=H^{-1} h$,

$$
\begin{aligned}
H & :=\left(\frac{1}{N} \sum_{j=1}^{N} \hat{A}_{j}^{\mathrm{T}} \hat{A}_{j}\right)-\overline{\hat{A}}^{\mathrm{T}} \overline{\hat{A}} \\
h & :=\left(\frac{1}{N} \sum_{j=1}^{N} \hat{A}_{j}^{\mathrm{T}}\left(\hat{q}_{j}-\hat{A}_{j} p^{*}\right)\right)-\overline{\hat{A}}^{\mathrm{T}} \hat{\bar{b}}
\end{aligned}
$$

\footnotetext{
${ }^{5}$ For even $k, \Gamma(k / 2)=(k / 2)$ !. For odd $k$, let $k^{\prime}=\frac{1}{2}(k-1)$, then $\Gamma(k / 2)=\Gamma\left(\frac{1}{2}+k^{\prime}\right)=\sqrt{\pi} \frac{\left(2 k^{\prime}+2\right) !}{(k+1) ! 4^{k^{\prime}+1}}$.
} 
where $\overline{\hat{A}}=\frac{1}{N} \sum_{j=1}^{N} \hat{A}_{j}$ and $\overline{\hat{b}}=\frac{1}{N} \sum_{j=1}^{N}\left(\hat{q}_{j}-\hat{A}_{j} p^{*}\right)$. Let $\tilde{A}_{j}=\hat{A}_{j}-A_{j}^{*}$ and $\tilde{q}_{j}=\hat{q}_{j}-q^{*}$. Then $H=H^{*}+\tilde{H}$ and $h=h^{*}+\tilde{h}$, with

$$
\begin{gathered}
H^{*}:=\left(\frac{1}{N} \sum_{j=1}^{N} A_{j}^{* \mathrm{~T}} A_{j}^{*}\right)-\overline{A^{*} \mathrm{~T}} \overline{A^{*}} \\
h^{*}:=\left(\frac{1}{N} \sum_{j=1}^{N} A_{j}^{* \mathrm{~T}}\left(q^{*}-A_{j}^{*} p^{*}\right)\right)-\overline{A^{*} \mathrm{~T}} \\
\tilde{H}=\frac{1}{N}\left[\sum_{j=1}^{N}\left(A_{j}^{*}+\tilde{A}_{j}\right)^{\mathrm{T}} \tilde{A}_{j}+\sum_{j=1}^{N} \tilde{A}_{j}^{\mathrm{T}} A_{j}^{*}\right]-\overline{A^{*} \mathrm{~T}} \overline{\tilde{A}}-\overline{\tilde{A}}^{\mathrm{T}} \overline{A^{*}}-\overline{\tilde{A}^{\mathrm{T}}} \overline{\tilde{A}} \\
\tilde{h}=\frac{1}{N}\left[\sum_{j=1}^{N}\left(A_{j}^{*}+\tilde{A}_{j}\right)^{\mathrm{T}}\left(\tilde{q}_{j}-\tilde{A}_{j} p^{*}\right)+\sum_{j=1}^{N} \tilde{A}_{j}^{\mathrm{T}}\left(q^{*}-A_{j}^{*} p^{*}\right)\right]-\overline{A^{*} \mathrm{~T}} \overline{\tilde{b}}-\overline{\tilde{A}}^{\mathrm{T}} \bar{b}^{*}-\overline{\tilde{A}^{\mathrm{T}}} \overline{\tilde{b}}
\end{gathered}
$$

where

$$
\begin{aligned}
\overline{A^{*}} & =\frac{1}{N} \sum_{j=1}^{N} A_{j}^{*} & \overline{b^{*}} & =\frac{1}{N} \sum_{j=1}^{N}\left(q^{*}-A_{j}^{*} p^{*}\right) \\
\overline{\tilde{A}} & =\frac{1}{N} \sum_{j=1}^{N} \tilde{A}_{j} & \overline{\tilde{b}} & =\frac{1}{N} \sum_{j=1}^{N}\left(\tilde{q}_{j}-\tilde{A}_{j} p^{*}\right)
\end{aligned}
$$

Notice that $H^{*}$ and $h^{*}$ depend only on $A_{j}^{*}, p^{*}$ and $q^{*}$. Moreover, since all functions in $L^{*}$ go through $\left(p^{*}, q^{*}\right)$, it must be that $p^{*}=\arg \min _{x} \operatorname{var} L^{*}(x)$, and thus $p^{*}=H^{*-1} h^{*}$. Rewriting $p^{\prime}=H^{-1} h$, gives $\left(H^{*}+\tilde{H}\right)\left(p^{*}+\left(p^{\prime}-p^{*}\right)\right)=h^{*}+\tilde{h}$. Applying $H^{*} p^{*}=h^{*}$ and solving for $p^{\prime}-p^{*}$ yields

$$
p^{\prime}-p^{*}=\left(H^{*}+\tilde{H}\right)^{-1}\left(\tilde{h}-\tilde{H} p^{*}\right)
$$

From the hypothesis it follows that $\left\|\tilde{A}_{j}\right\|<\Delta$ and $\left\|\tilde{q}_{j}\right\|<\Delta$. Applying these bounds and the properties of norms, it follows after some computation that

$$
\begin{aligned}
\|\tilde{H}\| & \leq 2 \Delta^{2}+\frac{4 \Delta}{N} \sum_{j=1}^{N}\left\|A_{j}^{*}\right\|, \\
\|\tilde{h}\| & \leq 2\left(1+\left\|p^{*}\right\|\right) \Delta^{2}+\frac{2 \Delta}{N} \sum_{j=1}^{N}\left[\left\|A_{j}^{*}\right\|\left(1+\left\|p^{*}\right\|\right)+\left\|q^{*}\right\|+\left\|A_{j}^{*}\right\|\left\|p^{*}\right\|\right] .
\end{aligned}
$$

Since $\Delta<\Delta_{0}$ and by definition $\Delta_{0} \leq \frac{1}{N} \sum_{j=1}^{N}\left\|A_{j}^{*}\right\|$, the above bounds may be further simplified to

$$
\begin{aligned}
\|\tilde{H}\| & <\left(\frac{6}{N} \sum_{j=1}^{N}\left\|A_{j}^{*}\right\|\right) \Delta \\
\|\tilde{h}\|< & <\left(\frac{2}{N} \sum_{j=1}^{N}\left[2\left\|A_{j}^{*}\right\|\left(1+\left\|p^{*}\right\|\right)+\left\|q^{*}\right\|+\left\|A_{j}^{*}\right\|\left\|p^{*}\right\|\right]\right) \Delta .
\end{aligned}
$$


Also by definition $\Delta_{0} \leq\left(\frac{12}{N}\left\|H^{*-1}\right\| \sum_{j=1}^{N}\left\|A_{j}^{*}\right\|\right)^{-1}$, so the bound in (27) can be simplified to $\|\tilde{H}\|<$ $\frac{1}{2\left\|H^{*-1}\right\|}$, and thus $\left\|H^{*-1} \tilde{H}\right\|<\frac{1}{2}$. From [19], if $M \in \mathbb{R}^{n \times n}$ and $\|M\|<1$, then $I-M$ is nonsingular and $\left\|(I-M)^{-1}\right\| \leq \frac{1}{1-\|M\|}$. Some computation using this fact and the bound on $\left\|H^{*-1} \tilde{H}\right\|$ provides

$$
\left\|\left(H^{*}+\tilde{H}\right)^{-1}\right\|<2\left\|H^{*-1}\right\| .
$$

So then

$$
\begin{aligned}
& \left\|p^{\prime}-p^{*}\right\| \leq\left\|\left(H^{*}+\tilde{H}\right)^{-1}\right\|\left\|\tilde{h}-\tilde{H} p^{*}\right\| \\
& \leq 2\left\|H^{*-1}\right\|\left(\|\tilde{h}\|+\|\tilde{H}\|\left\|p^{*}\right\|\right) \\
& <c_{2} \Delta \\
& c_{2}:=\frac{4\left\|H^{*-1}\right\|}{N} \sum_{j=1}^{N}\left(6\left\|A_{j}^{*}\right\|\left\|p^{*}\right\|+2\left\|A_{j}^{*}\right\|+\left\|q^{*}\right\|\right),
\end{aligned}
$$

which is the first part of the desired result. Applying this bound and the definition of $q^{\prime}$, we find after some computation that

$$
\begin{gathered}
\left\|q^{\prime}-q^{*}\right\|<c_{3} \Delta \\
\text { where } \quad c_{3}:=1+\frac{2 c_{2}}{N} \sum_{j=1}^{N}\left\|A_{j}^{*}\right\|
\end{gathered}
$$

which completes the desired result.

Proposition 1. Let

$$
\Delta_{0}^{m}=\min _{\substack{i \text { s.t. } \\ p_{i}^{*} \in P^{*}}}\left\{\frac{1}{N_{i}} \sum_{j=1}^{N_{i}}\left\|A_{i_{j}}^{*}\right\|, \quad\left(\frac{12}{N_{i}}\left\|H_{i}^{*-1}\right\| \sum_{j=1}^{N_{i}}\left\|A_{i_{j}}^{*}\right\|\right)^{-1}\right\},
$$

where $S_{i_{1}}^{*}, \ldots, S_{i_{N_{i}}}^{*}$ are the $N_{i} d$-simplices in $\operatorname{St}\left\{p_{i}^{*}\right\}$, and

$$
H_{i}^{*}=\left(\frac{1}{N_{i}} \sum_{j=1}^{N_{i}} A_{i_{j}}^{* \mathrm{~T}} A_{i_{j}}^{*}\right)-\left(\frac{1}{N_{i}} \sum_{j=1}^{N_{i}} A_{i_{j}}^{*}\right) \mathrm{T}\left(\frac{1}{N_{i}} \sum_{j=1}^{N_{i}} A_{i_{j}}^{*}\right) .
$$

We will examine the effect of a single iteration of the minvar algorithm on $p_{i} \in P, q_{i} \in Q$. The results will hold independently of $i$, giving the desired result.

The first stage of the minvar algorithm calculates the least squares projection $\Pi\left(\left.f_{\mathcal{P}}^{*}\right|_{S_{i}}\right)$ in each $d$-simplex $S_{i}$ of the approximation. (Step 2 of the algorithm. Since this proposition addresses the approximation version of the problem, there is no partitioning of data to be performed in Step 1.) Let $S_{i_{1}}, \ldots, S_{i_{N_{i}}}$ be the $d$-simplices in $\operatorname{St}\left\{p_{i}\right\}$. Since $f_{\mathcal{P}}^{*}$ and $f_{\mathcal{P}}$ are parameterized with the same abstract simplicial complex $\mathcal{S}^{*}$, $S_{i_{1}}^{*}, \ldots, S_{i_{N_{i}}}^{*}$ are the $d$-simplices in $\operatorname{St}\left\{p_{i}^{*}\right\}$. Let $\left.f_{\mathcal{P}}^{*}\right|_{S_{i_{j}}^{*}}(x)=A_{i_{j}}^{*}\left(x-p_{i}^{*}\right)+q_{i}^{*}$ and $\Pi\left(\left.f_{\mathcal{P}}^{*}\right|_{S_{i}}\right)=\hat{A}_{i_{j}}\left(x-p_{i}^{*}\right)+\hat{q}_{i_{j}}$. Since $\epsilon<\epsilon_{d} \leq \epsilon_{c}$, it follows from Lemma 1 that for each $j=1, \ldots, N_{i}$,

$$
\left\|\left[\begin{array}{c}
\hat{A}_{i_{j}}{ }^{\mathrm{T}}-A_{i_{j}}^{* \mathrm{~T}} \\
\hat{q}_{i_{j}}{ }^{\mathrm{T}}-q_{i}^{* \mathrm{~T}}
\end{array}\right]\right\|<c_{1, i_{j}} \epsilon^{2},
$$

where $c_{1, i_{j}}$ is given by (24). Let

$$
c_{1}=\max _{i=1, . . ., N} c_{1, i},
$$


where $N$ is the total number of $d$-simplices in $\mathcal{T}\left(P, \mathcal{S}^{*}\right)$. Then for $j=1 \ldots, N_{i}$,

$$
\left\|\left[\begin{array}{c}
\hat{A}_{i_{j}}^{\mathrm{T}}-A_{i_{j}}^{* \mathrm{~T}} \\
\hat{b}_{i_{j}}{ }^{\mathrm{T}}-b_{i_{j}}^{* \mathrm{~T}}
\end{array}\right]\right\|<c_{1} \epsilon^{2} .
$$

Step 3 of minvar moves the knots taking $\left(p_{i}, q_{i}\right) \rightarrow\left(p_{i}^{\prime}, q_{i}^{\prime}\right)$. Since $\epsilon<\epsilon_{d} \leq \sqrt{\Delta_{0}^{m} / c_{1}}$ by hypothesis, it follows that $c_{1} \epsilon^{2}<\Delta_{0}^{m}$. Thus, (34) implies that the bound in (12) is satisfied, permitting application of Lemma 2 , which gives

$$
\begin{aligned}
\left\|p_{i}^{\prime}-p_{i}^{*}\right\| & <c_{2, i} c_{1} \epsilon^{2}, \\
\left\|q_{i}^{\prime}-q_{i}^{*}\right\| & <c_{3, i} c_{1} \epsilon^{2},
\end{aligned}
$$

where

$$
\begin{aligned}
& c_{2, i}:=\frac{4\left\|H_{i}^{*-1}\right\|}{N_{i}} \sum_{j=1}^{N_{i}}\left(6\left\|A_{i_{j}}^{*}\right\|\left\|p_{i}^{*}\right\|+2\left\|A_{i_{j}}^{*}\right\|+\left\|q_{i}^{*}\right\|\right), \\
& c_{3, i}:=1+\frac{2 c_{2, i}}{N_{i}} \sum_{j=1}^{N_{i}}\left\|A_{i_{j}}^{*}\right\| .
\end{aligned}
$$

For each $p_{i}, q_{i}$ we can compute such bounds. Let

$$
\begin{aligned}
& c_{4}:=c_{1} \max _{i} c_{2, i} \\
& c_{5}:=c_{1} \max _{i} c_{3, i}
\end{aligned}
$$

Then, for all $i, p_{i}^{\prime}$ and $q_{i}^{\prime}$ satisfy

$$
\begin{aligned}
\left\|p_{i}^{\prime}-p_{i}^{*}\right\| & <c_{4} \epsilon^{2} \\
\left\|q_{i}^{\prime}-q_{i}^{*}\right\| & <c_{5} \epsilon^{2}
\end{aligned}
$$

which is the desired result.

\section{Proposition 2.}

First we establish by induction that for $k \geq 1$,

$$
\begin{aligned}
\frac{1}{c_{4}}\left(c_{4} \epsilon\right)^{2^{k}} & <\epsilon_{d}, \\
\left\|p_{i}^{(k)}-p_{i}^{*}\right\| & <\frac{1}{c_{4}}\left(c_{4} \epsilon\right)^{2^{k}}, \\
\left\|q_{i}^{(k)}-q_{i}^{*}\right\| & <\frac{c_{5}}{c_{4}}\left(c_{4} \epsilon\right)^{2^{k}} .
\end{aligned}
$$

For $k=1, c_{4} \epsilon^{2}<\epsilon_{d}$ since $\epsilon<\epsilon_{0} \leq \sqrt{\epsilon_{d} / c_{4}}$. For $k>1, \frac{1}{c_{4}}\left(c_{4} \epsilon\right)^{2^{k}}<\epsilon_{d}$ by the induction hypothesis. Moreover, $c_{4} \epsilon<1$ since $\epsilon<\epsilon_{0} \leq \frac{1}{c_{4}}$. Then $\frac{1}{c_{4}}\left(c_{4} \epsilon\right)^{2^{(k+1)}}=\left(\frac{1}{c_{4}}\left(c_{4} \epsilon\right)^{2^{k}}\right)\left(c_{4} \epsilon\right)^{2^{k}}<\epsilon_{d}$. This establishes (41). Since $\epsilon<\epsilon_{0} \leq \epsilon_{d}$, it follows that for $k=1$, after a single iteration of the minvar algorithm, (42) and (43) will hold by Proposition 1. For $k>1$, for all $i,\left\|p_{i}^{(k)}-p_{i}^{*}\right\|<\frac{1}{c_{4}}\left(c_{4} \epsilon\right)^{2^{k}}$ by the induction hypothesis. From (41), proven above, $\frac{1}{c_{4}}\left(c_{4} \epsilon\right)^{2^{k}}<\epsilon_{d}$. Since for all $i,\left\|p_{i}^{(k)}-p_{i}^{*}\right\|<\epsilon_{d}$, it follows from Proposition 1 that

$$
\begin{aligned}
&\left\|p_{i}^{(k+1)}-p_{i}^{*}\right\|<c_{4}\left(\frac{1}{c_{4}}\left(c_{4} \epsilon\right)^{2^{k}}\right)^{2}=\frac{1}{c_{4}}\left(c_{4} \epsilon\right)^{2^{(k+1)}} \\
&\left\|q_{i}^{(k+1)}-q_{i}^{*}\right\|<c_{5}\left(\frac{1}{c_{4}}\left(c_{4} \epsilon\right)^{2^{k}}\right)^{2}=\frac{c_{5}}{c_{4}}\left(c_{4} \epsilon\right)^{2^{(k+1)}},
\end{aligned}
$$

which establishes (42) and (43). Since $c_{4} \epsilon<1$, as argued above, it follows that (42) and (43) go to 0 as $k$ goes to infinity. 


\begin{tabular}{cccc} 
Vertices & uniform LS & minvar & minvar LS \\
\hline \hline $2^{2}$ & $1.79801 \mathrm{e}-01$ & $1.79818 \mathrm{e}-01$ & $1.79801 \mathrm{e}-01$ \\
$3^{2}$ & $9.85617 \mathrm{e}-02$ & $4.56123 \mathrm{e}-02$ & $4.48297 \mathrm{e}-02$ \\
$4^{2}$ & $4.45294 \mathrm{e}-02$ & $1.92465 \mathrm{e}-02$ & $1.89605 \mathrm{e}-02$ \\
$5^{2}$ & $2.47517 \mathrm{e}-02$ & $1.38427 \mathrm{e}-02$ & $1.36523 \mathrm{e}-02$ \\
$6^{2}$ & $1.55282 \mathrm{e}-02$ & $7.35190 \mathrm{e}-03$ & $7.25304 \mathrm{e}-03$ \\
$7^{2}$ & $1.05933 \mathrm{e}-02$ & $5.40420 \mathrm{e}-03$ & $5.34596 \mathrm{e}-03$ \\
$8^{2}$ & $7.63439 \mathrm{e}-03$ & $4.63040 \mathrm{e}-03$ & $4.56706 \mathrm{e}-03$ \\
$9^{2}$ & $5.84815 \mathrm{e}-03$ & $3.38636 \mathrm{e}-03$ & $3.34218 \mathrm{e}-03$ \\
$10^{2}$ & $4.53419 \mathrm{e}-03$ & $2.96688 \mathrm{e}-03$ & $2.92793 \mathrm{e}-03$ \\
$11^{2}$ & $3.71421 \mathrm{e}-03$ & $2.50778 \mathrm{e}-03$ & $2.47792 \mathrm{e}-03$ \\
$12^{2}$ & $2.99647 \mathrm{e}-03$ & $2.34302 \mathrm{e}-03$ & $2.32279 \mathrm{e}-03$ \\
$13^{2}$ & $2.49846 \mathrm{e}-03$ & $1.86386 \mathrm{e}-03$ & $1.84316 \mathrm{e}-03$
\end{tabular}

Table 1: RMSE of approximations by uniform LS, minvar, and minvar LS.

\section{$5 \quad$ Numerical Example}

This section presents an example of the minvar algorithm's performance on a "test function," $f:[0,1]^{2} \rightarrow \mathbb{R}^{2}$, given by

$$
f(x)=\left[\begin{array}{l}
\tanh \frac{5}{8}\left(2 x_{1}-4 x_{2}{ }^{4}+3 x_{2}{ }^{2}-1\right) \\
\tanh \frac{5}{8}\left(2 x_{1}{ }^{2}-x_{1}{ }^{4}+2 x_{2}-1\right)
\end{array}\right],
$$

which is invertible over the domain $D=[0,1]^{2}$. The implementation of minvar constructs an approximation to a discrete set of data, and includes constrained motion of boundary vertices as well as data dependent retriangulation. Since the test function is neither piecewise linear nor directly available, Theorem 1 provides no performance guarantees, but good performance under these circumstances suggests minvar's broader applicability. Two sets of numerical studies are presented. The first examines the effects of varying the number of vertices in the PL approximation, and the second examines how data set size affects approximations with a fixed number of vertices.

The first set of experiments fits PL approximations of differing sizes to a single data set. The data set was generated by sampling the test function on an $80 \times 80$ uniform grid over the domain. For each $n=2, \ldots, 13$, three different PL approximations with $n^{2}$ vertices were computed: i) the least squares continuous PL approximation on a fixed uniform triangulation of the domain (referred to as "uniform LS") ii) minvar, initialized on a uniform triangulation of the domain (referred to as "minvar"), and iii) the least squares continuous PL approximation on the final triangulation from minvar (referred to as "minvar LS"). Recall that when the domain triangulation is fixed, the least squares continuous PL approximation problem becomes linear-in-parameters and the solution can be computed directly [7,36]. Figure 2 shows the test function and several exemplars of the minvar approximations. Table 1 and Figure 3(a) show the root mean square error (RMSE) of the approximations as a function of the number of vertices. For $2^{2}$ domain vertices, all of them are on the corners of the domain and must remain fixed, so minvar can only change the range vertices. Since minvar is not guaranteed to give the least squares continuous PL approximation for a given triangulation, it is not surprising that the uniform LS approximation's RMSE is slightly lower than minvar's in this case. The RMSE difference between minvar and minvar LS approximations is less than $2 \%$. Least Squares could be applied as a post processing step to minvar, but since the differences are relatively small, this might not be necessary in application settings. From the triangulations of the minvar approximations in Figure 2, the domain triangulations move farther for lower numbers of vertices. As the number of vertices increases, the triangulations visually seem to deviate less from the initial uniform triangulation. The RMSE performance of minvar reflects this, giving the biggest reductions in RMSE as compared to uniform LS for triangulations with $3^{2}$ to $6^{2}$ vertices. Since this study uses initial conditions in which the vertices are 

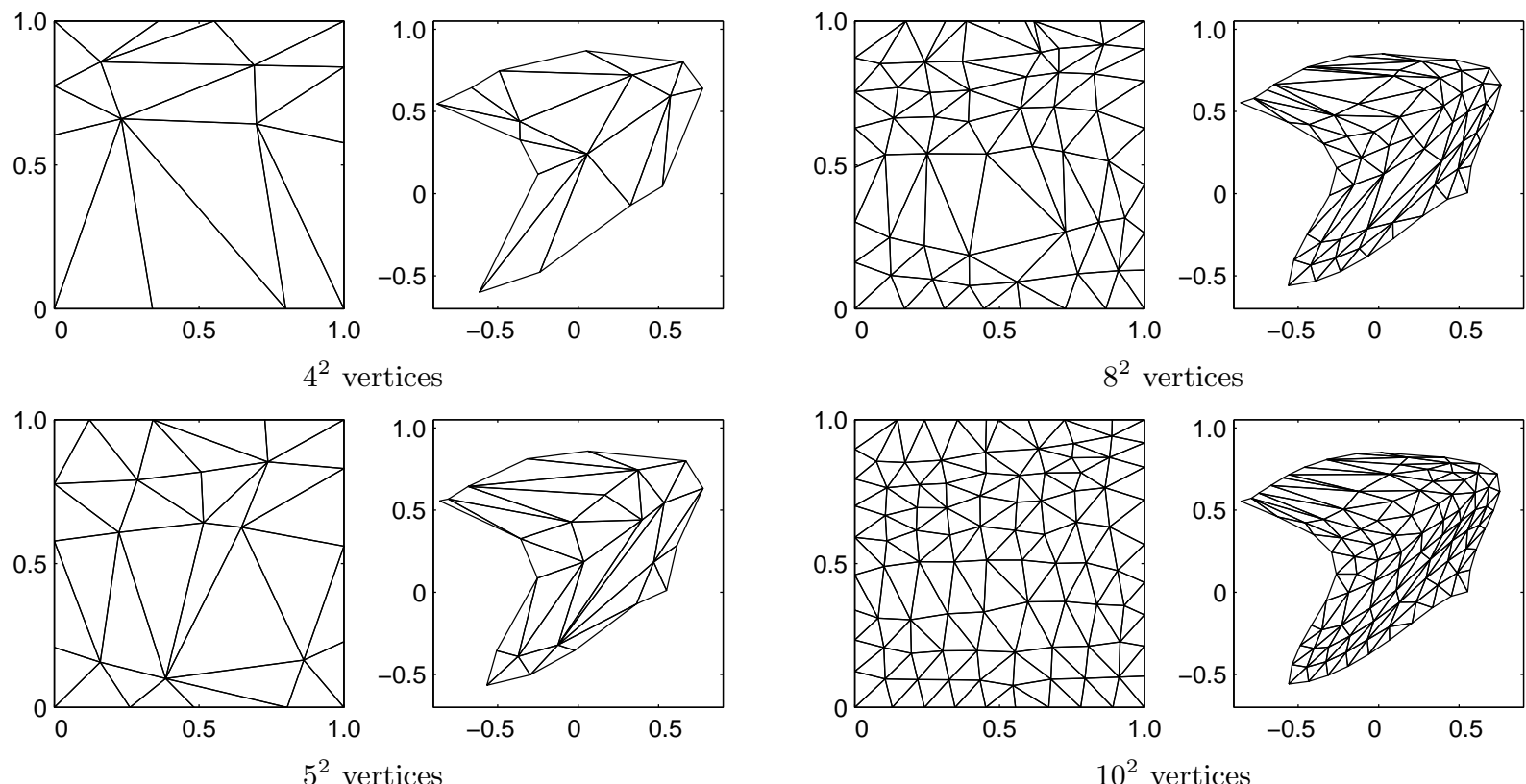

$5^{2}$ vertices
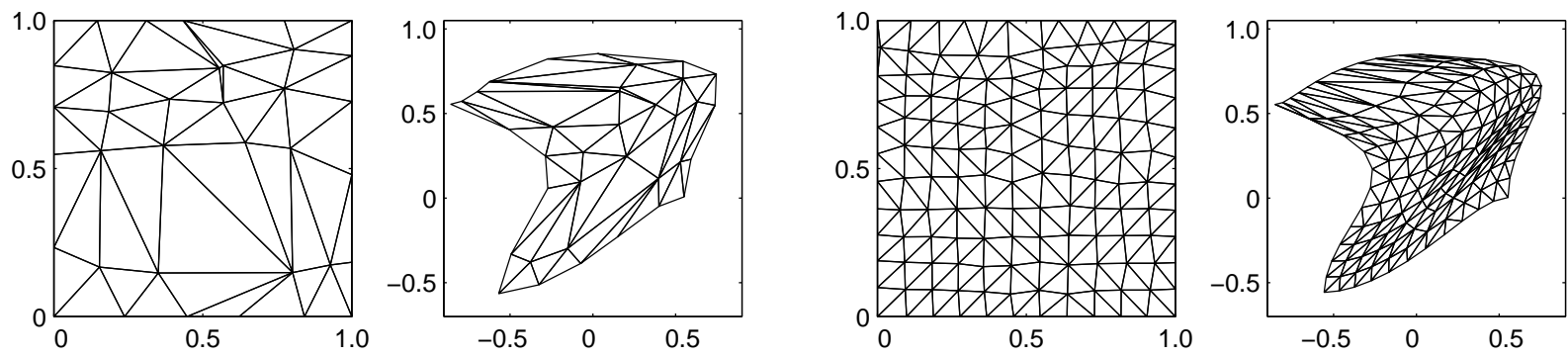

$6^{2}$ vertices

$12^{2}$ vertices
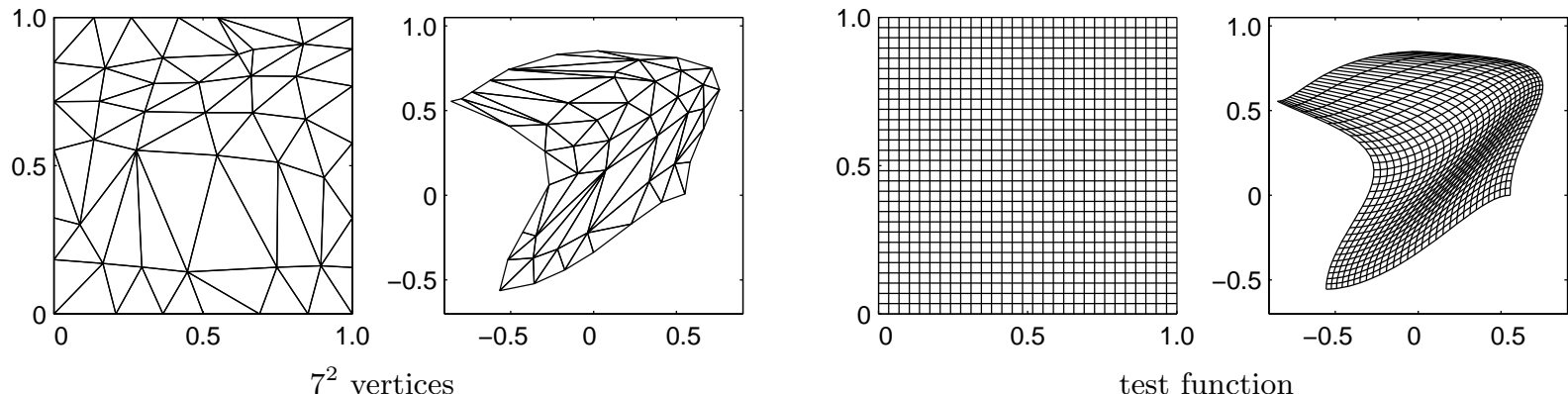

test function

Figure 2: Visualizations of the test function (lower-right) and a series of PL approximations to the test function. In each subfigure, the domain is displayed on the left and the range on the right. Note that the approximations are invertible, since there are no tangles in the range triangulations. 


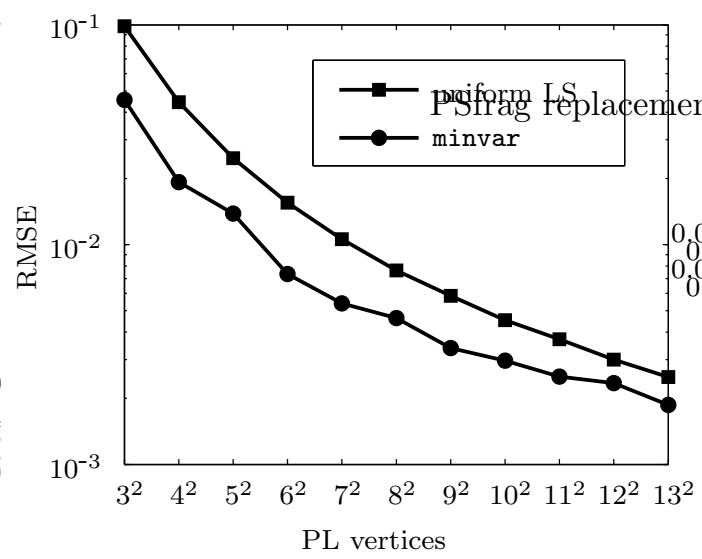

(a)

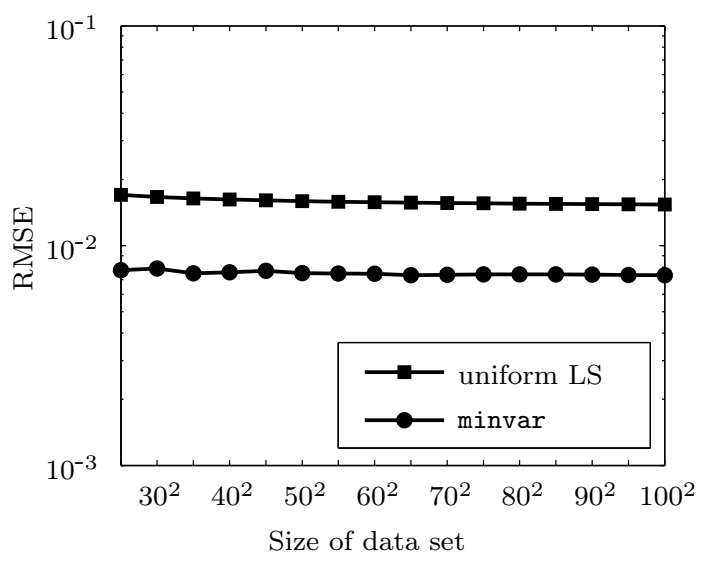

(b)

Figure 3: RMSE performance of minvar compared to a least squares continuous PL approximation on a uniform triangulation. (a) vertices in approximation vs. RMSE for approximations to the $80 \times 80$ data set. (b) density of training data set vs. RMSE on validation data for approximations with $6^{2}$ vertices.

on a uniform grid, approximations with large numbers of vertices may be getting caught in local minima near their initial conditions. In this case, performance could be improved by refining converged less dense approximations to create initial conditions for the dense approximations [40].

In the second set of experiments, PL approximations with $6^{2}$ vertices were trained using data sets of varying size. The PL approximations were chosen to have $6^{2}$ vertices because, as mentioned above, this is in the region of sizes where the performance gains from minvar are greatest. The data sets were generated by sampling the test function on a uniform $n \times n$ grid, $n=25,30,35, \ldots, 100$. The approximations were compared using a validation data set generated by evaluating the test function at 1000 points sampled from a uniform probability distribution over the domain. Figure 3(b) shows the validation set RMSE for minvar and uniform LS. With a $20 \times 20$ data set, minvar fails to run with a $6^{2}$ vertex approximation, because as the vertices move, several simplices shrink to the point that they do not contain enough data to make the linear least squares approximation unique. Data sparsity is a serious issue in this type of local approximation.

From this example, minvar shows marked benefit when the approximation has relatively few vertices relative to the complexity of the test function. We expect that minvar's performance on higher order (more vertices) approximations could be improved by seeding initial conditions based on lower order approximations. The algorithm produces a consistent approximation to variously sized data sets, so long as there is enough data for it to run.

\section{Conclusion}

Numerous applications require the simultaneous approximation of a function and its inverse from a set of discrete data. While there is a substantial literature on function approximation, very little addresses the constraint of invertibility. The inverse of a continuous piecewise linear function can be computed in closed form, which is ideal for applications requiring the approximation of a function and its inverse. In the PL literature, the partition often fixed, in which case the minimum squared error approximation problem is linear-in-parameters. The problem becomes nonlinear-in-parameters when the domain partition is allowed to move.

The minvar algorithm is a novel method for computing continuous PL approximations to data. Rather than using gradient descent on the parameters, minvar takes advantage of the structure of PL functions, iteratively moving the vertices of the approximation based on local least squares fits. The minvar algo- 
rithm is proven to converge locally in the special case that the data generating function is itself PL and available directly rather than through discrete data. While this result seems very natural, complexity in the proof arises from the interaction of the domain triangulations of the data generating function and its approximation. Indeed, many difficulties in constructing PL approximations, such as triangulation tangles, arise primarily from the combinatorial properties of PL functions. For general approximation problems, this added complexity may cause PL approximation to appear less attractive than other nonlinear-in-parameters approximation techniques, but for an important subset of applications PL's closed form invertibility makes the combinatorial complexity cost effective.

The present work can be extended in several directions. The analysis here addresses the approximation rather than estimation version of the problem. A formal connection between the approximation and estimation versions could be constructed in the appropriate statistical framework. Similarly, there is no study of the effects of noise on the convergence properties of minvar. Since data from the data generating function are only used for computing the least squares approximations, and least squares has good noise properties, the authors expect that the minvar algorithm will also have good noise properties. The present analytical work only considers piecewise linear data generating functions. A desirable extension would be to show that the algorithm converges to the locally best PL approximation, given that one exists. For scalar functions there are generalized convexity conditions that characterize existence and uniqueness of (possibly discontinuous) piecewise linear approximations $[4,18,26]$, but the authors are unaware of similar results for dimensions greater than one or with continuous piecewise approximations. Numerical experience suggests that minvar does have good convergence properties on other types of functions. Practical application of minvar raises a number of challenging issues such as constrained motion of vertices on the boundary of the domain, methods to avoid and correct triangulation tangling, and retriangulation or adaptation of the combinatorial parameters of the PL approximation. Due to space limitations, these topics will be addressed in detail in a subsequent paper on the use of minvar in engineering applications.

\section{A Geometric Properties of Simplices and Triangulations}

Proving properties of the minvar algorithms requires some insight into the underlying geometric structures upon which PL functions are constructed. This appendix presents a number of geometry facts and proofs upon which the proof of convergence relies.

\section{A.1 Barycentric Coordinates as Distances}

It is often convenient to represent points in $\mathbb{R}^{d}$ using barycentric coordinates with respect to the vertices of some $d$-simplex. Let $p_{1}, \ldots, p_{d+1}$ be affinely independent points in $\mathbb{R}^{d}$. Let $x \in \mathbb{R}^{d}$ and let $\bar{\alpha}=\left[\begin{array}{lll}\alpha_{1} & \cdots & \alpha_{d+1}\end{array}\right]^{\mathrm{T}}$ be such that $x=\sum_{i=1}^{d+1} \alpha_{i} p_{i}$ and $\sum_{i=1}^{d+1} \alpha_{i}=1 . \quad \bar{\alpha}$ are called the barycentric coordinates of $x$ with respect to $p_{1}, \ldots, p_{d+1}$. If $\bar{\alpha} \in \Delta_{d+1}:=\left\{\bar{\alpha} \in \mathbb{R}^{d+1} \mid \alpha_{i} \geq 0, \sum_{i=1}^{d+1} \alpha_{i}=1\right\}$, then $x \in \operatorname{conv}\left(p_{1}, \ldots, p_{d+1}\right)$, and $\Delta_{d}$ is called the standard $d$-simplex.

The distance between a point $x \in \mathbb{R}^{d}$ and a nonempty set $A \subseteq \mathbb{R}^{d}$ is well defined [42] and written as $\delta(x, A):=\inf _{z \in A}\|x-z\|$. Let $H_{i}=\operatorname{aff}\left(\left\{p_{1}, \ldots, p_{d+1}\right\}-\left\{p_{i}\right\}\right)$, the hyperplane opposing $p_{i}$. Let $\left(a_{i}, c_{i}\right)$ be an implicit representation for $H_{i}$, that is, $H_{i}=\left\{z \in \mathbb{R}^{d} \mid a_{i}{ }^{\mathrm{T}} z+c_{i}=0\right\}$. The distance of a point $x \in \mathbb{R}^{d}$ to $H_{i}$ is given by

$$
\delta\left(x, H_{i}\right)=\frac{\left|a_{i}{ }^{\mathrm{T}} x+c_{i}\right|}{\left\|a_{i}\right\|} .
$$

We define $\delta_{s}\left(x, H_{i}\right)$ as the signed distance of $x$ from $H_{i}$. That is, $\left|\delta_{s}\left(x, H_{i}\right)\right|=\delta\left(x, H_{i}\right)$, and $\delta_{s}\left(x, H_{i}\right)>0$ for $x$ on the same side of $H_{i}$ as $p_{i}$, and $\delta_{s}\left(x, H_{i}\right)<0$ for $x$ on the opposite side of $H_{i}$ as $p_{i}$.

By the following claim, the barycentric coordinates of $x$ can be interpreted as the scaled distances of $x$ from hyperplanes $H_{1}, \ldots, H_{d+1}$. 
Claim 1. Let $x \in \mathbb{R}^{d}$. Let $\bar{\alpha}=\left[\begin{array}{lll}\alpha_{1} & \cdots & \alpha_{d+1}\end{array}\right]^{\mathrm{T}}$ be the barycentric coordinates of $x$ with respect to the affinely independent points $p_{1}, \ldots, p_{d+1} \in \mathbb{R}^{d}$. Then $\delta_{s}\left(x, H_{i}\right)=\alpha_{i} \delta\left(p_{i}, H_{i}\right)$.

Proof. First we establish that $\delta\left(x, H_{i}\right)=\left|\alpha_{i}\right| \delta\left(p_{i}, H_{i}\right)$, and then show that the sign of $\alpha_{i}$ indicates the side of $H_{i}$ on which $p_{i}$ lies, giving the desired result. Let $\left(a_{i}, c_{i}\right)$ be an implicit representation of $H_{i}$. Then

$$
\begin{aligned}
\delta\left(x, H_{i}\right) & =\frac{\left|a_{i}{ }^{\mathrm{T}} x+c_{i}\right|}{\left\|a_{i}\right\|} \\
& =\frac{1}{\left\|a_{i}\right\|}\left|a_{i}{ }^{\mathrm{T}}\left(\sum_{j=1}^{d+1} \alpha_{j} p_{j}\right)+c_{i}\right| \\
& =\left|\sum_{j=1}^{d+1} \alpha_{j}\left(\frac{a_{i}^{\mathrm{T}} p_{j}+c_{i}}{\left\|a_{i}\right\|}\right)\right|
\end{aligned}
$$

For $j \neq i, p_{j} \in H_{i}$, which implies that $a_{i}{ }^{\mathrm{T}} p_{j}+c_{i}=0$. It follows that

$$
\delta\left(x, H_{i}\right)=\left|\alpha_{i}\right| \delta\left(p_{i}, H_{i}\right) .
$$

Since $i$ ) for a given $x, \alpha_{i}=0$ if and only if $x \in H_{i}$, ii) for $x=p_{i}, \alpha_{i}=1$, and iii) $\alpha_{i}$ is an affine function of $x$, it follows that $\alpha_{i}>0$ for $x$ on the same side of $H_{i}$ as $p_{i}$, and $\alpha_{i}<0$ for $x$ on the opposite side of $H_{i}$ as $p_{i}$. Thus, $\delta_{s}\left(x, H_{i}\right)=\alpha_{i} \delta\left(p_{i}, H_{i}\right)$.

Claim 2. Let $x \in \mathbb{R}^{d}$. Let $\bar{\alpha}=\left[\begin{array}{lll}\alpha_{1} & \cdots & \alpha_{d+1}\end{array}\right]^{\mathrm{T}}$ be barycentric coordinates of $x$ with respect to the affinely independent points $p_{1}, \ldots, p_{d+1}$. The distance from $x$ to the affine subspace $A=\operatorname{aff}\left(\left\{p_{1}, \ldots p_{k}\right\}\right)$, is given by $\delta(x, A)=\bar{\alpha}_{s}^{\mathrm{T}} G \bar{\alpha}_{s}$, where $\bar{\alpha}_{s}=\left[\begin{array}{llll}\alpha_{k+1} & \alpha_{k+2} & \cdots & \alpha_{d+1}\end{array}\right]^{\mathrm{T}}$ and $G \in \mathbb{R}^{(d-k+1) \times(d-k+1)}$, defined in (46), is a positive definite matrix whose entries depend only on $p_{1}, \ldots, p_{k}$.

Proof. The distance of a point to a linear subspace can be computed using Gram determinants [30]. A translation by $p_{k}$ turns the affine subspace $A$ into a linear subspace. Let $\tilde{A}=A-p_{k}$. For $i=1, \ldots, d+1$, let $\tilde{p}_{i}=p_{i}-p_{k}$. Let $\tilde{x}=x-p_{k}$, and note that

$$
\tilde{x}=\left(\sum_{i=1}^{d+1} \alpha_{i} p_{i}\right)-p_{k}=\sum_{i=1}^{d+1} \alpha_{i}\left(p_{i}-p_{k}\right)=\sum_{i=1}^{d+1} \alpha_{i} \tilde{p}_{i} .
$$

Let

$$
\begin{gathered}
g(y, z)=\left|\begin{array}{ccccc}
\tilde{p}_{1}^{\mathrm{T}} \tilde{p}_{1} & \tilde{p}_{2}{ }^{\mathrm{T}} \tilde{p}_{1} & \cdots & \tilde{p}_{k-1}{ }^{\mathrm{T}} \tilde{p}_{1} & y^{\mathrm{T}} \tilde{p}_{1} \\
\tilde{p}_{1}{ }^{\mathrm{T}} \tilde{p}_{2} & \tilde{p}_{2}{ }^{\mathrm{T}} \tilde{p}_{2} & & \tilde{p}_{k-1}{ }^{\mathrm{T}} \tilde{p}_{2} & y^{\mathrm{T}} \tilde{p}_{2} \\
\vdots & & & & \vdots \\
\tilde{p}_{1}^{\mathrm{T}} \tilde{p}_{k-1} & \tilde{p}_{2}{ }^{\mathrm{T}} \tilde{p}_{k-1} & & \tilde{p}_{k-1}{ }^{\mathrm{T}} \tilde{p}_{k-1} & y^{\mathrm{T}} \tilde{p}_{k-1} \\
\tilde{p}_{1}{ }^{\mathrm{T}} z & \tilde{p}_{2}{ }^{\mathrm{T}} z & \cdots & \tilde{p}_{k-1}{ }^{\mathrm{T}} z & y^{\mathrm{T}} z
\end{array}\right|, \\
b=\left|\begin{array}{cccc}
\tilde{p}_{1}{ }^{\mathrm{T}} \tilde{p}_{1} & \tilde{p}_{2}{ }^{\mathrm{T}} \tilde{p}_{1} & \cdots & \tilde{p}_{k-1}{ }^{\mathrm{T}} \tilde{p}_{1} \\
\tilde{p}_{1}^{\mathrm{T}} \tilde{p}_{2} & \tilde{p}_{2}{ }^{\mathrm{T}} \tilde{p}_{2} & & \tilde{p}_{k-1}{ }^{\mathrm{T}} \tilde{p}_{2} \\
\vdots & & & \vdots \\
\tilde{p}_{1}{ }^{\mathrm{T}} \tilde{p}_{k-1} & \tilde{p}_{2}{ }^{\mathrm{T}} \tilde{p}_{k-1} & \cdots & \tilde{p}_{k-1}{ }^{\mathrm{T}} \tilde{p}_{k-1}
\end{array}\right| .
\end{gathered}
$$

The quantity $g(y, y)$ is the Gram determinant for $\tilde{p}_{1}, \ldots, \tilde{p}_{k-1}, y$, while $b$ is the Gram determinant for $\tilde{p}_{1}, \ldots, \tilde{p}_{k-1}$. The squared distance from $x$ to $A, \delta^{2}(x, A)$, is given by the ratio of Gram determinants

$$
\delta^{2}(x, A)=\delta^{2}(\tilde{x}, \tilde{A})=\frac{g(\tilde{x}, \tilde{x})}{b} .
$$


Using $(45), \delta^{2}(x, A)$ can be rewritten as

$$
\delta^{2}(x, A)=\frac{1}{b} \sum_{i=1}^{d+1} \sum_{j=1}^{d+1} g\left(\tilde{p}_{i}, \tilde{p}_{j}\right) \alpha_{i} \alpha_{j}
$$

Notice that $g$ is symmetric in its arguments, $g(y, z)=g(z, y)$. Also, for $1 \leq i \leq k, g\left(\tilde{p}_{i}, y\right)=0$. Thus

$$
\delta^{2}(x, A)=\frac{1}{b} \sum_{i=k+1}^{d+1} \sum_{j=k+1}^{d+1} g\left(\tilde{p}_{i}, \tilde{p}_{j}\right) \alpha_{i} \alpha_{j}=\bar{\alpha}_{s}^{\mathrm{T}} G \bar{\alpha}_{s}
$$

where $G$ is given by

$$
G=\frac{1}{b}\left[\begin{array}{cccc}
g\left(\tilde{p}_{k+1}, \tilde{p}_{k+1}\right) & g\left(\tilde{p}_{k+2}, \tilde{p}_{k+1}\right) & \cdots & g\left(\tilde{p}_{d+1}, \tilde{p}_{k+1}\right) \\
g\left(\tilde{p}_{k+1}, \tilde{p}_{k+2}\right) & & & g\left(\tilde{p}_{d+1}, \tilde{p}_{k+2}\right) \\
\vdots & & & \vdots \\
g\left(\tilde{p}_{k+1}, \tilde{p}_{d+1}\right) & g\left(\tilde{p}_{k+2}, \tilde{p}_{d+1}\right) & \cdots & g\left(\tilde{p}_{d+1}, \tilde{p}_{d+1}\right)
\end{array}\right] .
$$

It remains to show that $G$ is positive definite. Consider $x$ such that $\alpha_{i} \neq 0$, for some $i=k+1, \ldots, d+1$. By the previous claim, $x$ must lie some nonzero distance away from $H_{i}$. Since $A \subseteq H_{i}, \delta(x, A)>0$. Thus, $G$ must be positive definite.

\section{A.2 Properties of the Dilation}

The dilation arises in Lemma 1 in measuring how the approximation's mismatched triangulation affects the least squares fits. First general properties of the dilation are presented, and then the specific result needed for Lemma 1 is presented.

Claim 3. Let $S \subseteq \mathbb{R}^{d}$ be a d-simplex with vertices $p_{1}, \ldots, p_{d+1}$. Let

$$
\epsilon_{\min }=-\left(\sum_{i=1}^{d+1} 1 / \delta\left(p_{i}, H_{i}\right)\right)^{-1}
$$

where $H_{i}$ is the opposing hyperplane to $p_{i}$, as defined above. $\mathcal{D}\left(S, \epsilon_{\min }\right)$ is a single point. For $\epsilon>\epsilon_{\min }, \mathcal{D}(S, \epsilon)$ is a d-simplex, with faces parallel to and translated distance $|\epsilon|$ away from the faces of $S$. For $\epsilon_{\min } \leq \epsilon \leq 0$, $\mathcal{D}(S, \epsilon) \subseteq S$, while for $\epsilon \geq 0, S \subseteq \mathcal{D}(S, \epsilon)$.

Proof. Recall that the definition of the dilation is

$$
\mathcal{D}(S, \epsilon)=\left\{x=\sum_{i=1}^{d+1} \alpha_{i} p_{i} \mid \sum_{i=1}^{d+1} \alpha_{i}=1, \forall i, \alpha_{i} \geq \frac{-\epsilon}{\delta\left(p_{i}, H_{i}\right)}\right\},
$$

First we will show that $\mathcal{D}\left(S, \epsilon_{\min }\right)$ is a single point. Note that

$$
\sum_{i=1}^{d+1} \frac{-\epsilon_{\min }}{\delta\left(p_{i}, H_{i}\right)}=1
$$

and any point in in $\mathcal{D}\left(S, \epsilon_{\min }\right)$ has $\alpha_{i} \geq-\epsilon_{\min } / \delta\left(p_{i}, H_{i}\right)$ for all $i$. It follows that $\mathcal{D}\left(S, \epsilon_{\min }\right)$ is a single point.

For $\epsilon>\epsilon_{\min }, \mathcal{D}\left(S, \epsilon_{\min }\right)$ can be viewed in light of Claim 1 as the intersection of $d+1$ closed half-spaces, with the boundary hyperplane of each half-spaces is parallel to a respective $H_{i}$. Moreover the intersection is nonempty, since each of the half-spaces contains $\mathcal{D}\left(S, \epsilon_{\min }\right)$, and bounded, since the $\alpha_{i}$ 's are bounded. By Theorem 3.2.5 of [42], a polyhedral set (intersection of closed half spaces) is a polytope (convex hull of a 
finite number of points) if and only if the set is bounded. Thus $\mathcal{D}\left(S, \epsilon_{\min }\right)$ is a polytope. Since $\mathcal{D}\left(S, \epsilon_{\min }\right)$ is the intersection of $d+1$ closed halfspaces, it can have at most $\left(\begin{array}{c}d+1 \\ d\end{array}\right)=d+1$ vertices. For $\epsilon>\epsilon_{\min }$, inspection of the $\alpha_{i}$ 's shows that $\mathcal{D}\left(S, \epsilon_{\min }\right)$ is a $d$-dimensional set, in which case $\mathcal{D}\left(S, \epsilon_{\min }\right)$ must have at least $d+1$ vertices. Thus $\mathcal{D}\left(S, \epsilon_{\min }\right)$ is a simplex.

That $\mathcal{D}(S, \epsilon) \subseteq S$ for $\epsilon_{\min } \leq \epsilon \leq 0$ and $S \subseteq \mathcal{D}(S, \epsilon)$ for $\epsilon \geq 0$ follows from the definition of the dilation and Claim 1.

The proof of Lemma 1 requires a relationship between incident $d$-simplices.

Claim 4. Let $S_{a}, S_{b} \subseteq \mathbb{R}^{d}$ be incident d-simplices, that is $S_{a} \cap S_{b}=s_{a b}$, where $s_{a b} \leq S_{a}, S_{b}$ is a (k-1)simplex, $1 \leq k<d-1$. Let vert $S_{a}=\left\{p_{1}, \ldots, p_{d+1}\right\}$, vert $S_{b}=\left\{p_{1}, \ldots, p_{k}, q_{k+1}, \ldots, q_{d+1}\right\}$, and vert $s_{a b}=$ $\left\{p_{1}, \ldots, p_{k}\right\}$. Let $A=$ aff $s_{a b}$. Then $\exists \kappa_{a, b}>0$ such that $\forall \epsilon>0$, if $x \in \mathcal{D}\left(S_{a}, \epsilon\right) \cap S_{b}$ then $\delta(x, A)<\kappa_{a, b} \epsilon$.

Proof. Let

$$
\begin{gathered}
V_{1}=\left[\begin{array}{ccc}
p_{1} & \cdots & p_{k} \\
1 & \cdots & 1
\end{array}\right] \quad V_{3}=\left[\begin{array}{ccc}
p_{k+1} & \cdots & p_{d+1} \\
1 & \cdots & 1
\end{array}\right] \quad V_{2}=\left[\begin{array}{ccc}
q_{k+1} & \cdots & q_{d+1} \\
1 & \cdots & 1
\end{array}\right] \\
V_{a}=\left[\begin{array}{ll}
V_{1} & V_{3}
\end{array}\right] \quad V_{b}=\left[\begin{array}{ll}
V_{1} & V_{2}
\end{array}\right]
\end{gathered}
$$

The barycentric coordinates of $x \in \mathbb{R}^{d}$ with respect to $S_{a}$ and $S_{b}$ are given by, respectively,

$$
\left[\begin{array}{lll}
\alpha_{1} & \cdots & \alpha_{d+1}
\end{array}\right]^{\mathrm{T}}=V_{a}^{-1}\left[\begin{array}{l}
x \\
1
\end{array}\right] \quad\left[\begin{array}{lll}
\beta_{1} & \cdots & \beta_{d+1}
\end{array}\right]^{\mathrm{T}}=V_{b}^{-1}\left[\begin{array}{l}
x \\
1
\end{array}\right]
$$

For $x \in \mathcal{D}\left(S_{a}, \epsilon\right)$, the barycentric coordinates with respect to $S_{a}$ must by definition satisfy

$$
\left[\begin{array}{lll}
\alpha_{1} & \cdots & \alpha_{d+1}
\end{array}\right]^{\mathrm{T}} \geq\left[\begin{array}{lll}
-\epsilon / \delta\left(p_{1}, H_{1}\right) & \cdots & -\epsilon / \delta\left(p_{d+1}, H_{d+1}\right)
\end{array}\right]^{\mathrm{T}}
$$

For $x \in S_{b}$, the barycentric coordinates of $x$ with respect to $S_{b}$ must satisfy

$$
\left[\begin{array}{lll}
\beta_{1} & \cdots & \beta_{d+1}
\end{array}\right]^{\mathrm{T}} \geq\left[\begin{array}{lll}
0 & \cdots & 0
\end{array}\right]^{\mathrm{T}}
$$

We are interested in $x \in \mathcal{D}\left(S_{a}, \epsilon\right) \cap S_{b}$, so both (48) and (49) must hold. The barycentric coordinates of $x$ with respect to $S_{a}$ and $S_{b}$ are related by

$$
\left[\begin{array}{lll}
\alpha_{1} & \cdots & \alpha_{d+1}
\end{array}\right]^{\mathrm{T}}=V_{a}^{-1} V_{b}\left[\begin{array}{lll}
\beta_{1} & \cdots & \beta_{d+1}
\end{array}\right]^{\mathrm{T}}
$$

Because $V_{a}$ and $V_{b}$ have the first $k$ columns in common, $V_{a}{ }^{-1} V_{b}$ has the structure

$$
V_{a}^{-1} V_{b}=\left[\begin{array}{ll}
I & M_{1} \\
0 & M_{2}
\end{array}\right]
$$

where $M_{1} \in \mathbb{R}^{k \times(d+1-k)}$ and $M_{2} \in \mathbb{R}^{(d+1-k) \times(d+1-k)}$.

The vertices of $S_{b}$ are partitioned into two sets, $\sigma_{1}=\left\{p_{1}, \ldots, p_{k}\right\}$ and $\sigma_{2}=\left\{q_{k+1}, \ldots, q_{d+1}\right\}$. Any point $x \in S_{b}$ can be described as a triplet $\left(t, \beta^{1}, \beta^{2}\right)$, where $t \in[0,1], \beta^{1} \in \Delta_{k}$, and $\beta^{2} \in \Delta_{d+1-k}$. $\beta^{1}$ and $\beta^{2}$ are barycentric coordinates corresponding to $\sigma_{1}$ and $\sigma_{2}$, representing points in conv $\sigma_{1}$ and conv $\sigma_{2}$ respectively. The parameter $t$ is the scaled distance of $x$ along the line between the point in conv $\sigma_{1}$ and conv $\sigma_{2}$. This representation is unique for $x \in S-\left(\operatorname{conv} \sigma_{1} \cup \operatorname{conv} \sigma_{2}\right)$. The relationship between $x$ and $\left(t, \beta^{1}, \beta^{2}\right)$ is given by

$$
\left[\begin{array}{ll}
x^{\mathrm{T}} & 1
\end{array}\right]^{\mathrm{T}}=(1-t) V_{1} \beta^{1}+t V_{3} \beta^{2} .
$$

The relationship between the barycentric coordinates of $x$ with respect to $S_{b}$ and the triplet $\left(t, \beta^{1}, \beta^{2}\right)$ is

$$
\left[\begin{array}{c}
\beta_{1} \\
\vdots \\
\beta_{k} \\
\beta_{k+1} \\
\vdots \\
\beta_{d+1}
\end{array}\right]=(1-t)\left[\begin{array}{c}
\beta_{1}^{1} \\
\vdots \\
\beta_{k}^{1} \\
0 \\
\vdots \\
0
\end{array}\right]+t\left[\begin{array}{c}
0 \\
\vdots \\
0 \\
\beta_{1}^{2} \\
\vdots \\
\beta_{d+1-k}^{2}
\end{array}\right]
$$


and $\left(t, \beta^{1}, \beta^{2}\right)$ may be computed from $\beta$ by

$$
t=\sum_{i=k+1}^{d+1} \beta_{i} \quad \beta_{i}^{1}=\beta_{i} /(1-t) \quad \beta_{i}^{2}=\beta_{i+k} / t
$$

Rewriting the right-hand side of (50) using this representation gives

$$
\left[\begin{array}{lll}
\alpha_{1} & \cdots & \alpha_{d+1}
\end{array}\right]^{\mathrm{T}}=(1-t)\left[\begin{array}{l}
I \\
0
\end{array}\right]\left[\begin{array}{lll}
\beta_{1}^{1} & \cdots & \beta_{k}^{1}
\end{array}\right]^{\mathrm{T}}+s\left[\begin{array}{l}
M_{1} \\
M_{2}
\end{array}\right]\left[\begin{array}{lll}
\beta_{1}^{2} & \cdots & \beta_{d+1-k}^{2}
\end{array}\right]^{\mathrm{T}} .
$$

The desired result will be achieved by bounding $\sum_{i=k+1}^{d+1} \beta_{i}$ using the following two subclaims and then applying Claim 2. The proofs of the subclaims follow the present proof.

Subclaim 1. Let $\zeta$ be given by

$$
\begin{aligned}
\zeta & =-\max _{\beta^{2} \in \Delta_{d+1-k}} f\left(\beta^{2}\right) \\
f\left(\beta^{2}\right) & =\min _{1 \leq i \leq d+1-k}\left[M_{2} \beta^{2}\right]_{i} \delta\left(p_{i+k}, H_{i+k}\right),
\end{aligned}
$$

where $[\cdot]_{i}$ is the $i^{\text {th }}$ vector component. Then $\zeta$ is well defined and $\zeta>0$.

Subclaim 2. Let $x \in S_{b}$ have representation $\left(\beta^{1}, \beta^{2}, t\right)$. If $t>\frac{1}{\zeta} \epsilon$, then there exists $i$ such that $\alpha_{i}<$ $-\epsilon / \delta\left(p_{i}, H_{i}\right)$.

Let $\epsilon>0$ be given. Let $x \in \mathcal{D}\left(S_{a}, \epsilon\right) \cap S_{b}$. It follows that the barycentric representation of $x$ with respect to $S_{a}$ satisfies $\alpha_{i} \geq-\epsilon / \delta\left(p_{i}, H_{i}\right)$ for all $i$. By the contrapositive of Subclaim 2 , the representation of $x$ as $\left(t, \beta^{1}, \beta^{2}\right)$ must have $t \leq \frac{1}{\zeta} \epsilon$. It follows that the barycentric coordinates of $x$ with respect to $S_{b}$ satisfy

$$
\sum_{i=k+1}^{d+1} \beta_{i}=t \leq \frac{1}{\zeta} \epsilon
$$

Let $\bar{\beta}_{s}^{\mathrm{T}}=\left[\begin{array}{lll}\beta_{k+1} & \cdots & \beta_{d+1}\end{array}\right]^{\mathrm{T}}$. By Claim 2 and the properties of symmetric positive definite matrices,

$$
\delta(x, A)=\sqrt{\bar{\beta}_{s} G \bar{\beta}_{s}} \leq \sqrt{\lambda_{\max }(G)}\left\|\bar{\beta}_{s}\right\|_{2}
$$

The infinity norm dominates the 2 norm, and since $x \in S_{b}, \beta_{i} \geq 0$, so

$$
\delta(x, A) \leq \sqrt{\lambda_{\max }(G)} \sum_{i=k+1}^{d+1} \beta_{i}
$$

Finally, applying (53) gives

$$
\delta(x, A) \leq \frac{\sqrt{\lambda_{\max }(G)}}{\zeta} \epsilon
$$

which is the desired result, with $c=\frac{\sqrt{\lambda_{\max }(G)}}{\zeta}$.

Subclaim 1. First we show that $M_{2} \beta^{2}$ has a strictly negative component $\forall \beta^{2} \in \Delta_{d+1-k}$. Let $\beta^{2}$ be given. Let $\beta^{1} \in \Delta_{k}$ be

$$
\beta^{1}=\left[\begin{array}{lll}
1 / k & \cdots & 1 / k
\end{array}\right]^{\mathrm{T}}
$$

For $t=0$, the point with representation $\left(t, \beta^{1}, \beta^{2}\right)$ has barycentric coordinates with respect to $S_{a}$ given by

$$
\left[\begin{array}{llllll}
\alpha_{1} & \cdots & \alpha_{k} & \alpha_{k+1} & \cdots & \alpha_{d+1}
\end{array}\right]^{\mathrm{T}}=\left[\begin{array}{llllll}
1 / k & \cdots & 1 / k & 0 & \cdots & 0
\end{array}\right]^{\mathrm{T}}
$$


For any point with $0<t \leq 1$, the point with representation $\left(t, \beta^{1}, \beta^{2}\right)$ lies strictly outside of $S_{a}$. Thus, in the barycentric coordinates of this point with respect to $S_{a}$, there exists $i$ such that $\alpha_{i}<0$. Consider a point given by $\left(t, \beta^{1}, \beta^{2}\right)$ with $t>0$, but very small, then

$$
\left[\alpha_{1} \cdots \alpha_{k} \alpha_{k+1} \cdots \alpha_{d+1}\right]^{\mathrm{T}}=(1-t)\left[\frac{1}{k} \cdots \frac{1}{k} 0 \cdots 0\right]^{\mathrm{T}}+t\left[\left(M_{1} \beta^{2}\right)^{\mathrm{T}}\left(M_{2} \beta^{2}\right)^{\mathrm{T}}\right]^{\mathrm{T}} .
$$

For $t$ very small, the first $k$ components of $\alpha$ must be positive by continuity. Since some component $\alpha_{i}$ must be strictly negative when $t>0$, it must be that a component of $t M_{2} \beta^{2}$ is strictly negative. Since $t$ is positive, it follows that a component of $M_{2} \beta^{2}$ is strictly negative.

Since $\delta\left(p_{i+k}, H_{i+k}\right)>0$ for $1 \leq i \leq d+1-k$, and at least one component of $M_{2} \beta^{2}$ is strictly negative, it follows that $f\left(\beta^{2}\right)<0$ for all $\beta^{2}$. Since sum, multiplication, and minimum are continuous, $f$ is a continuous function. Since $\Delta_{d+1-k}$ is compact, $f$ must take it's maximum for some $\beta^{2}$, thus $\zeta$ is well defined. As we argued above, $f\left(\beta^{2}\right)<0$ for all $\beta^{2}$, thus

$$
\max _{\beta^{2} \in \Delta_{d+1-k}} f\left(\beta^{2}\right)<0
$$

Thus $\zeta>0$.

Subclaim 2. By the definition of $\zeta$ there exists $i$ such that $\left[M_{2} \beta^{2}\right]_{i} \delta\left(p_{i+k}, H_{i+k}\right) \leq-\zeta$ and by hypothesis $t>\frac{1}{\zeta} \epsilon$. Thus

$$
\begin{aligned}
\alpha_{k+i} & =t\left[M_{2} \beta^{2}\right]_{i} \\
& <-\frac{1}{-\zeta} \epsilon \frac{-\zeta}{\delta\left(p_{i+k}, H_{i+k}\right)} \\
& =-\frac{\epsilon}{\delta\left(p_{i+k}, H_{i+k}\right)},
\end{aligned}
$$

Thus, there exist $i$ such that $\alpha_{i}<\epsilon / \delta\left(p_{i}, H_{i}\right)$.

\section{A.3 Properties of Triangulations}

The conditions for the parameters to generate a geometric simplicial complex are proven.

Claim 5. $\mathcal{K}(P, \mathcal{S})$ is a geometric simplicial complex if

1. $\forall \alpha \in \mathcal{S}, P(\alpha)$ are affinely independent

2. $s_{1}, s_{2} \in \mathcal{K}(P, \mathcal{S}) \Longrightarrow s_{1} \cap s_{2} \leq s_{1}, s_{2}$.

Moreover, if these properties hold, then $\operatorname{vert}(\mathcal{K}(P, \mathcal{S}))=P$.

Proof. Let $s_{1} \in \mathcal{K}(P, \mathcal{S})$ and let $s_{2} \leq s_{1}$. Since $s_{1} \in \mathcal{K}(P, \mathcal{S})$, there exists $\alpha_{1}$ such that $s_{1}=\operatorname{conv} P\left(\alpha_{1}\right)$. By Property $1, P\left(\alpha_{1}\right)$ is affinely independent, thus vert $s_{1}=P\left(\alpha_{1}\right)$. Since $s_{2} \leq s_{1}$, vert $s_{2} \subseteq$ vert $s_{1}$. Since $\mathcal{S}$ is an abstract complex, $\exists \alpha_{2} \in \mathcal{S}$ such that $\alpha_{2} \subseteq \alpha_{1}$ and $P\left(\alpha_{2}\right)=$ vert $s_{2}$. Thus $s_{2} \in \mathcal{K}(P, \mathcal{S})$. Thus, $s_{1} \in \mathcal{K}$ and $s_{2} \leq s_{1} \Longrightarrow s_{2} \in \mathcal{K}$. Since $\mathcal{K}(P, \mathcal{S})$ is a collection of simplices satisfying this and Property 2 , it follows that $\mathcal{K}(P, \mathcal{S})$ is a geometric simplicial complex.

Since the vertex set of $\mathcal{S}$ is $\{1, \ldots, n\}$, it follows from the definition of $\mathcal{K}(\cdot, \cdot)$ that $\left\{p_{i}\right\} \in \mathcal{K}(P, \mathcal{S})$ for $i=1, \ldots, n$, and moreover these are the only singleton sets in $\mathcal{K}(P, \mathcal{S})$. If follows that vert $(\mathcal{K}(P, \mathcal{S}))=P$.

\section{A.4 Properties of PL functions}

Claims pertaining to the the parameterization, continuity, and invertibility of PL functions are provided below. 
Claim 6. Let $f_{\mathcal{P}}$ be a continuous piecewise linear function parameterized by $(P, Q, \mathcal{S})$. Let $S_{i}, S_{j} \in \mathcal{T}(P, \mathcal{S})$ be such that $S_{i} \cap S_{j} \neq \emptyset$. Let $S_{i} \cap S_{j}$ be a $(k-1)$-simplex, so then $S_{i}$ and $S_{j}$ share $k$ vertices in common. Let vert $S_{i}=\left\{p_{i_{1}}, \ldots, p_{i_{d+1}}\right\}$, vert $S_{j}=\left\{p_{i_{1}}, \ldots, p_{i_{k}}, p_{j_{k+1}}, \ldots, p_{j_{d+1}}\right\}$, and vert $S_{i} \cap S_{j}=\left\{p_{i_{1}}, \ldots, p_{i_{k}}\right\}$. Then, 1. $f_{\mathcal{P}}$ in $S_{i}$ and $S_{j}$ is given by

$$
\begin{array}{rlrl}
\left.f_{\mathcal{P}}\right|_{S_{i}}(x) & =U_{i} V_{i}^{-1}\left[\begin{array}{ll}
x^{\mathrm{T}} & 1
\end{array}\right]^{\mathrm{T}} & & \left.f_{\mathcal{P}}\right|_{S_{j}}(x)=U_{j} V_{j}^{-1}\left[\begin{array}{llll}
x^{\mathrm{T}} & 1
\end{array}\right]^{\mathrm{T}} \\
U_{i} & =\left[\begin{array}{lll}
q_{i_{1}} & \cdots & q_{i_{d+1}}
\end{array}\right] & U_{j} & =\left[\begin{array}{llllll}
q_{i_{1}} & \cdots & q_{i_{k}} & q_{j_{k+1}} & \cdots & q_{j_{d+1}}
\end{array}\right] \\
V_{i} & =\left[\begin{array}{ccc}
p_{i_{1}} & \cdots & p_{i_{d+1}} \\
1 & & 1
\end{array}\right] & V_{j}=\left[\begin{array}{cccccc}
p_{i_{1}} & \cdots & p_{i_{k}} & p_{j_{k+1}} & \cdots & p_{j_{d+1}} \\
1 & & 1 & 1 & & 1
\end{array}\right]
\end{array}
$$

Then $U_{i} V_{i}^{-1}\left[\begin{array}{ll}x^{\mathrm{T}} & 1\end{array}\right]^{\mathrm{T}}=U_{j} V_{j}^{-1}\left[\begin{array}{ll}x^{\mathrm{T}} & 1\end{array}\right]^{\mathrm{T}}$ for $x \in \operatorname{aff}\left(S_{i} \cap S_{j}\right)$.

2. In nonhomogeneous form, let $\left.f_{\mathcal{P}}\right|_{S_{i}}(x)=A_{i} x+b_{i}$ and $\left.f_{\mathcal{P}}\right|_{S_{j}}(x)=A_{j} x+b_{j}$. Let $L$ be the linear subspace parallel to aff $\left(S_{i} \cap S_{j}\right)$. Then $L \subseteq \mathcal{N}\left(A_{i}-A_{j}\right)$.

Proof. Notice that $V_{i}^{-1}\left[\begin{array}{ll}x^{\mathrm{T}} & 1\end{array}\right]^{\mathrm{T}}$ and $V_{j}^{-1}\left[\begin{array}{ll}x^{\mathrm{T}} & 1\end{array}\right]^{\mathrm{T}}$ are the barycentric coordinates of $x$ with respect to $S_{i}$ and $S_{j}$, respectively. For $x \in \operatorname{aff}\left(S_{i} \cap S_{j}\right)$, it follows from Claim 2 that the last $d+1-k$ components of $V_{i}^{-1}\left[\begin{array}{ll}x^{\mathrm{T}} & 1\end{array}\right]^{\mathrm{T}}$ and $V_{j}^{-1}\left[\begin{array}{ll}x^{\mathrm{T}} & 1\end{array}\right]^{\mathrm{T}}$ must be 0 . Moreover, since the first $k$ columns of $V_{i}$ and $V_{j}$ are identical, $V_{i}^{-1}\left[\begin{array}{ll}x^{\mathrm{T}} & 1\end{array}\right]^{\mathrm{T}}=V_{j}^{-1}\left[\begin{array}{ll}x^{\mathrm{T}} & 1\end{array}\right]^{\mathrm{T}}$ for $x \in \operatorname{aff}\left(S_{i} \cap S_{j}\right)$. It follows that $U_{i} V_{i}^{-1}\left[\begin{array}{ll}x^{\mathrm{T}} & 1\end{array}\right]^{\mathrm{T}}=U_{j} V_{j}^{-1}\left[\begin{array}{ll}x^{\mathrm{T}} & 1\end{array}\right]^{\mathrm{T}}$ for $x \in \operatorname{aff}\left(S_{i} \cap S_{j}\right)$.

Since the above result holds independently of the representation of the affine function, $A_{i} x+b_{i}=A_{j} x+b_{j}$ for $x \in \operatorname{aff}\left(S_{i} \cap S_{j}\right)$. Let $x_{0} \in S_{i} \cap S_{j}$, and let $y_{0}=A_{i} x_{0}+b_{i}=A_{j} x_{0}+b_{j}$. Then $A_{i}\left(x-x_{0}\right)+y_{0}=A_{j}\left(x-x_{0}\right)+y_{0}$, or equivalently $\left(A_{i}-A_{j}\right)\left(x-x_{0}\right)=0$, for $x \in \operatorname{aff}\left(S_{i} \cap S_{j}\right)$. It follows that $L \subseteq \mathcal{N}\left(A_{i}-A_{j}\right)$, where $L$ is the vector space parallel to aff $\left(S_{i} \cap S_{j}\right)$.

Claim 7 (Continuity in vertices). Consider two continuous piecewise linear functions, $f_{\mathcal{P}}^{*}$ parameterized by $\mathcal{P}^{*}=\left(P^{*}, Q^{*}, \mathcal{S}^{*}\right)$ and $f_{\mathcal{P}}$ parameterized by $\left(P, Q, \mathcal{S}^{*}\right)$, such that $\left|\mathcal{T}\left(P, \mathcal{S}^{*}\right)\right|=\left|\mathcal{T}\left(P^{*}, \mathcal{S}^{*}\right)\right|$. Let c $>0$. There exists $c^{\prime}=c^{\prime}\left(\mathcal{P}^{*}, c\right)$ such that, for $0<\epsilon<r_{3}$, where

$$
r_{3}=\min _{S^{*} \in \mathcal{T}\left(P^{*}, \mathcal{S}^{*}\right)} \sup \left\{\epsilon\left|\mathcal{D}\left(S^{*}, \epsilon\right) \subseteq\right| \mathrm{ClSt} S^{*} \mid\right\} .
$$

If $\left\|p_{i}-p_{i}^{*}\right\|<\epsilon$ and $\left\|q_{i}-q_{i}^{*}\right\|<c \epsilon$ for all $i$, then

$$
\left\|f_{\mathcal{P}}-f_{\mathcal{P}}^{*}\right\|_{\infty}<c^{\prime} \epsilon
$$

where $c^{\prime}$ is given by (55). That is, a continuous PL function is continuous in its vertices.

Proof. Let $0<\epsilon<r_{3}$ be given. Let $\left.f_{\mathcal{P}}^{*}\right|_{S_{i}^{*}}(x)=A_{i}^{*} x+b_{i}^{*}$ and $\left.f_{\mathcal{P}}\right|_{S_{i}}(x)=A_{i} x+b_{i}$. For any $j$ such that $p_{j}^{*} \in \operatorname{vert} S_{i}^{*}, q_{j}^{*}=A_{i}^{*} p_{j}^{*}+b_{i}^{*}$. Thus we can write $\left.f_{\mathcal{P}}^{*}\right|_{S_{i}^{*}}(x)=A_{i}^{*}\left(x-p_{j}^{*}\right)+q_{j}^{*}$. Similarly we can write $\left.f_{\mathcal{P}}\right|_{S_{i}}(x)=A_{i}\left(x-p_{j}\right)+q_{j}$ for any $j$ such that $p_{j} \in \operatorname{vert} S_{i}$.

The $\infty$ norm can be broken down as follows.

$$
\left\|f_{\mathcal{P}}-f_{\mathcal{P}}^{*}\right\|_{\infty}=\max _{i=1, \ldots, N}\left\|\left.\left(f_{\mathcal{P}}-f_{\mathcal{P}}^{*}\right)\right|_{S_{i}}\right\|_{\infty}
$$

Let $\varphi_{i}(x)=A_{i}^{*}\left(x-p_{j}^{*}\right)+q_{j}^{*}$, where $p_{j}^{*} \in \operatorname{vert} S_{i}^{*}$. Let $\psi_{i}(x)=f_{\mathcal{P}}^{*}(x)-\varphi_{i}(x)$. Then,

$$
\begin{aligned}
\left\|\left.\left(f_{\mathcal{P}}-f_{\mathcal{P}}^{*}\right)\right|_{S_{i}}\right\|_{\infty} & =\left\|\left.\left(f_{\mathcal{P}}-\varphi_{i}-\psi_{i}\right)\right|_{S_{i}}\right\|_{\infty} \\
& \leq\left\|\left.\left(f_{\mathcal{P}}-\varphi_{i}\right)\right|_{S_{i}}\right\|_{\infty}+\left\|\left.\psi_{i}\right|_{S_{i}}\right\|_{\infty}
\end{aligned}
$$

These terms will be individually bounded. Since $S_{i}$ is convex, and $\left\|\left(f_{\mathcal{P}}-\varphi_{i}\right)(x)\right\|_{2}$ is the square root of a positive semi-definite quadratic function, and thus a convex function on $S_{i}$, it will take its maximum at a 
vertex of $S_{i}$ :

$$
\begin{aligned}
\left\|\left.\left(f_{\mathcal{P}}-\varphi_{i}\right)\right|_{S_{i}}\right\|_{\infty} & =\max _{p_{j} \in \operatorname{vert} S_{i}}\left\|A_{i}\left(p_{j}-p_{j}\right)+q_{j}-\left[A_{i}^{*}\left(p_{j}-p_{j}^{*}\right)+q_{j}^{*}\right]\right\| \\
& \leq \max _{p_{j} \in \operatorname{vert} S_{i}}\left\|q_{j}-q_{j}^{*}\right\|+\left\|A_{i}^{*}\right\|\left\|p_{j}-p_{j}^{*}\right\| \\
& <\left(c+\left\|A_{i}^{*}\right\|\right) \epsilon
\end{aligned}
$$

It remains to bound $\left\|\left.\psi_{i}\right|_{S_{i}}\right\|_{\infty}$. By definition $\psi_{i}$ is a continuous piecewise linear function and $\left.\psi_{i}\right|_{S_{i}^{*}} \equiv 0$. For $0<\epsilon<r_{3}, \mathcal{D}\left(S_{i}^{*}, \epsilon\right) \cap S_{k}^{*} \neq \emptyset$ if and only if $S_{k}^{*} \in \mathrm{St} S_{i}^{*}$ by the definition of $r_{3}$. Since $\left\|p_{i}-p_{i}^{*}\right\|<\epsilon$ for all $i$, then $S_{i} \subseteq \mathcal{D}\left(S_{i}^{*}, \epsilon\right)$. Using these facts,

$$
\left\|\left.\psi_{i}\right|_{S_{i}}\right\|_{\infty}=\max _{S_{k}^{*} \in \operatorname{St} S_{i}^{*}}\left\|\left.\psi_{i}\right|_{S_{i} \cap S_{k}^{*}}\right\|_{\infty} .
$$

Let $S_{k}^{*} \in \mathrm{St} S_{i}^{*}$ and let $x_{k} \in \operatorname{aff}\left(S_{k}^{*} \cap S_{i}^{*}\right)$. Then $\left.\psi_{i}\right|_{S_{k}^{*}}(x)=\left(A_{k}^{*}-A_{i}^{*}\right)\left(x-x_{k}\right)$. By Claim $6,\left(A_{k}^{*}-A_{i}^{*}\right)\left(x-x_{k}\right)=$ 0 for $x \in \operatorname{aff}\left(S_{k}^{*} \cap S_{i}^{*}\right)$. Thus, for $x \in S_{i} \cap S_{k}^{*},\left\|\psi_{i}(x)\right\| \leq\left\|A_{k}^{*}-A_{i}^{*}\right\| \delta\left(x, \operatorname{aff}\left(S_{k}^{*} \cap S_{i}^{*}\right)\right)$. But $x \in S_{k}^{*} \cap \mathcal{D}\left(S_{i}^{*}, \epsilon\right)$, so by Claim $4, \delta\left(x, \operatorname{aff}\left(S_{k}^{*} \cap S_{i}^{*}\right)\right)<\kappa_{i, k} \epsilon$. Thus,

$$
\left\|\left.\psi_{i}\right|_{S_{i}}\right\|_{\infty} \leq\left[\max _{S_{k}^{*} \in \operatorname{St} S_{i}^{*}}\left\|A_{k}^{*}-A_{i}^{*}\right\| \kappa_{i, k}\right] \epsilon
$$

It follows that

$$
\left\|\left.\left(f_{\mathcal{P}}-f_{\mathcal{P}}^{*}\right)\right|_{S_{i}}\right\|_{\infty}<\left(c+\left\|A_{i}^{*}\right\|+\max _{S_{k}^{*} \in \operatorname{St} S_{i}^{*}}\left\|A_{k}^{*}-A_{i}^{*}\right\| \kappa_{i, k}\right) \epsilon .
$$

Thus $\left\|f_{\mathcal{P}}-f_{\mathcal{P}}^{*}\right\|_{\infty}<c^{\prime} \epsilon$, where

$$
c^{\prime}=\max _{i}\left(c+\left\|A_{i}^{*}\right\|+\max _{S_{k}^{*} \in \operatorname{St} S_{i}^{*}}\left\|A_{k}^{*}-A_{i}^{*}\right\| \kappa_{i, k}\right) .
$$

Claim 8. Let $f_{\mathcal{P}}$ be a piecewise linear function parameterized by $\mathcal{P}=(P, Q, \mathcal{S})$. If $\mathcal{T}(Q, \mathcal{S})$ is also a triangulation, then the piecewise linear function is invertible on its range, and the inverse, $f_{\mathcal{P}}{ }^{-1}$, is parameterized by $\mathcal{P}^{-1}=(Q, P, \mathcal{S})$.

Proof. Let $f_{\mathcal{P}}(x)=f_{\mathcal{P}}(y)$ for $x, y \in \mathcal{T}(P, \mathcal{S})$. Since $\mathcal{T}(Q, \mathcal{S})$ is also a triangulation, there exists a unique $s \in \mathcal{T}(Q, \mathcal{S})$ such that $f_{\mathcal{P}}(x) \in s$ and $s \leq s^{\prime}$ for all other $s^{\prime} \in \mathcal{T}(Q, \mathcal{S})$ such that $f(x) \in s^{\prime}$. Let vert $s=$ $\left\{q_{j_{1}}, \ldots, q_{j_{k}}\right\}$. Let $\bar{s} \in \mathcal{T}(P, \mathcal{S})$ with vert $\bar{s}=\left\{p_{j_{1}}, \ldots, p_{j_{k}}\right\}$. Then it follows that $x, y \in \bar{s}$. Let $S_{i}$ be a $d$-simplex such that $\bar{s} \leq S_{i}$. Since $\mathcal{T}(Q, \mathcal{S})$ is a triangulation, $f_{\mathcal{P}}\left(S_{i}\right)$ is also a $d$-simplex, and hence the linear part of $\left.f_{\mathcal{P}}\right|_{S_{i}}$ must be full rank. It follows that $x=y$. Thus $f_{\mathcal{P}}$ is one-to-one, and hence invertible on its range.

It may be shown that the inverse is parameterized by $\mathcal{P}^{-1}=(Q, P, \mathcal{S})$ through arithmetic manipulation of (2).

\section{References}

[1] C. G. Atkeson, A. W. Moore, And S. SchaAl, Locally weighted learning, Artificial Intelligence Review, 11 (1997), pp. 11-73.

[2] M. J. BAINES, Algorithms for optimal discontinuous piecewise linear and constant $L_{2}$ fits to continuous functions with adjustable nodes in one and two dimensions, Mathematics of Computation, 62 (1994), pp. 645-669. 
[3] A. R. BARRon, Universal approximation bounds for superpositions of a sigmoidal function, IEEE Transactions on Information Theory, 39 (1993), pp. 930-945.

[4] D. L. Barrow, C. K. Chui, P. W. Smith, And J. D. Ward, Unicity of best mean approximation by second order splines with variable knots, Mathematics of Computation, 32 (1978), pp. 1131-1143.

[5] K. Q. Brown, Voronoi diagrams from convex hulls, Information Processing Letters, 9 (1979), pp. 223228.

[6] E. W. Cheney, Introduction to Approximation Theory, McGraw-Hill Book Co., 1966.

[7] C. K. Chui, Multivariate splines, Society for Industrial and Applied Mathematics, 1988.

[8] N. J. Cowan, J. D. Weingarten, And D. E. KoditscheK, Visual servoing via navigation functions, IEEE Transactions on Robotics and Automation, (2002). (accepted).

[9] P. J. DAvis, Interpolation and Approximation, Dover Publications, Inc., 1975.

[10] C. De Boor, K. Höllig, and S. Riemenschneider, Box Splines, Springer-Verlag, 1993.

[11] N. Dyn, D. LEvin, AND S. RIPPA, Algorithms for the construction of data dependent triangulations, in Algorithms for Approximation, II, Chapman and Hall, 1990, pp. 185-192.

[12] — Data dependent triangulations for piecewise linear interpolation, IMA Journal of Numerical Analysis, 10 (1990), pp. 137-54.

[13] H. Edelsbrunner, Geometry and Topology for Mesh Generation, Cambridge University Press, 2001.

[14] H. Edelsbrunner AND N. SHAh, Incremental topological flipping works for regular triangulations, Algorithmica, 15 (1996), pp. 223-241.

[15] S. FioRI, Probability density function learning by unsupervised neurons, International Journal of Neural Systems, (2001), pp. 399-417.

[16] J. H. Friedman, Multivariate adaptive regression splines, The Annals of Statistics, 19 (1991), pp. 1141.

[17] S. FURRY AND J. KAINZ, Rapid algorithm development applied to engine management systems, in Proc. 1998 SAE Intl Congress \& Exposition, 1998.

[18] R. C. Gayle And J. M. Wolfe, Unicity in piecewise polynomial $L_{1}$-approximation via an algorithm, Mathematics of Computation, 65 (1996), pp. 647-660.

[19] G. E. Golub And C. F. Van Loan, Matrix Computations, John Hopkins University Press, 1996.

[20] R. E. Groff, Training Piecewise Linear Homeomorphisms for the Approximation of Maps with Known Invariants, $\mathrm{PhD}$ thesis, University of Michigan, 2002. in preparation.

[21] R. E. Groff, P. P. Khargonekar, D. E. Koditschek, T. T. Thieret, and L. Mestha, Modeling and control of color xerographic processes, in Proceedings of 38th IEEE Conference on Decision and Control, vol. 2, 1999, pp. 1697-1702.

[22] R. E. Groff, D. E. Koditschek, And P. P. Khargonekar, Piecewise linear homeomorphisms: the scalar case, in Proceedings of the International Joint Conference on Neural Ne tworks, 2000.

[23] R. E. Groff, D. E. Koditschek, P. P. Khargonekar, and T. T. Thieret, Representation of color space transformations for effective calibration and control, in IS\&T's NIP16: International Conference on Digital Printing Technology, 2000, pp. 255-260. 
[24] N. HAI, J. NI, AND J. YUAN, Generalized model formulation technique for error synthesis and error compensation on machine tools, in Proceedings of the NAMRX XXVI Conference, Society of Manufacturing Engineers, 1998.

[25] J. B. Kioustelidis, Optimal segmented approximations, Computing, 24 (1980), pp. 1-8.

[26] — O Optimal segmented polynomial $L_{s}$-approximations, Computing, 26 (1981), pp. 239-246.

[27] D. E. KoditscheK And E. Rimon, Robot navigation functions on manifolds with boundary, Advances in Applied Mathematics, 11 (1990), pp. 412-442.

[28] C. L. Lawson, Properties of n-dimensional triangulations, Computer Aided Geometric Design, 3 (1986), pp. 231-246.

[29] G. G. Lorentz, Approximation of Functions, Holt, Rhinehart and Winston, 1966.

[30] D. G. Luenberger, Optimization by Vector Space Methods, John Wiley \& Sons, Inc., 1969.

[31] F. P. Preparata And M. I. Shamos, Computational geometry : an introduction, Springer-Verlag, 1985.

[32] M. H. RAIBERT, Legged Robots That Balance, MIT Press, 1986.

[33] E. Rimon And D. E. Koditschek, The construction of analytic diffeomorphisms for exact robot navigation on star worlds, Transactions of the American Mathematical Society, 327 (1991), pp. 71-115.

[34] — Exact robot navigation using artificial potential fields, IEEE Transactions on Robotics and Automation, 8 (1992), pp. 501-518.

[35] W. J. Schwind And D. E. Koditschek, Approximating the stance map of a 2 dof monoped runner, Journal of Nonlinear Science, 10 (2000), pp. 533-568.

[36] E. V. Shikin And A. I. Plis, Handbook on Splines for the User, CRC Press, 1995.

[37] E. H. Spanier, Algebraic Topology, McGraw-Hill, 1966.

[38] M. SpIVAK, Calculus on Manifolds, Perseus Books, 1965.

[39] C. J. Stone, M. H. Hansen, C. Kooperberg, and Y. K. Truong, Polynomial splines and their tensor products in extended linear modeling, The Annals of Statistics, 25 (1997), pp. 1371-1470.

[40] Y. Tourigny And M. J. BAines, Analysis of an algorithm for generating locally optimal meshes for $L_{2}$ approximation by discontinuous piecewise polynomials, Mathematics of Computation, 66 (1997), pp. 623-650.

[41] Y. TOurigny and F. Hülsemann, A new moving mesh algorithm for the finite element solution of variational problems, SIAM J. Numer. Anal., 35 (1998), pp. 1416-1438.

[42] R. Webster, Convexity, Oxford University Press, 1994.

[43] J. C. Ziegert And P. DAtseris, Basic considerations for robot calibration, International Journal of Robotics and Automation, 4 (1989), pp. 158-66. 